\title{
The Regenerating Forest of Magbukún Aeta in Morong, Bataan, Philippines: A Biological Hotspot for Protection and Conservation
}

\author{
Dean Carlo F. Galias ${ }^{1,2^{*}}$ And Virginia C. Cuevas ${ }^{2}$
}

\begin{abstract}
This research focused on the study of a tropical semi-evergreen rain forest community structure within the ancestral domain of the Aetas in Morong, Bataan. Point-Centered Quarter Method (PCQM) was used where five $200 \mathrm{~m}$ transects were laid across a 28-ha study site. Trees with $\geq 1 \mathrm{~cm}$ diameter at $1.3 \mathrm{~m}$ from the ground (diameter at breast height $[\mathrm{DBH}]$ ) were sampled. Trees encountered were categorized into four groups: seedlings, saplings, pole trees and standard trees based on girth classes. A total of 76 tree species belonging to 61 genera and 35 families were recorded. The most represented family was Moraceae, followed by Dipterocarpaceae, Sapindaceae and Lauraceae. The species with highest importance percentage (IP) were Shorea contorta Vidal (Dipterocarpaceae) (IP=9.731), Macaranga tanarius (L.) Muell.-Arg. (Euphorbiaceae) $(\mathrm{IP}=5.659)$ and Canarium europhyllum $\mathrm{G}$. Perkins (Burseraceae) $(\mathrm{IP}=4.538)$. Very high values of species diversity were calculated $\left(H^{\prime}=3.817 ; D^{\prime}=0.968\right)$. The area was previously a dipterocarp forest and can now be classified as a young regenerating forest, composed primarily of seedlings many of which are dipterocarps. There were 17 threatened species, including two critically endangered and two endangered species (listed in The IUCN Red List of Threatened Species (2017-3) and DAO (2017-11) as well as twelve endemic species identified as present in the area. This forest is a biological hotspot that needs utmost protection and conservation.
\end{abstract}

KEYWORDS: Ancestral domain, Endemic, PCQM, Threatened species, Tree diversity

\section{INTRODUCTION}

The Philippine forestlands provide various ecosystem services including biodiversity maintenance, carbon sequestration, timber and non-timber forest products (Arnaiz, 2012), watershed services, and landscape beauty (Calderon et al., 2013). They also supply numerous environmental, economic and social services, all of which are significant to human development (PTFCF, 2013). In addition, approximately $30 \%$ of the population, including some 12 to 15 million indigenous peoples, are dependent on forests for their survival and cultural needs (DENR 2009, as cited by PTFCF, 2013).

The forests of Morong, Bataan in Luzon have long been the

${ }^{1}$ Department of Biology, College of Science, Bicol University, Legazpi City, Philippines

${ }^{2}$ Institute of Biological Sciences, College of Arts and Sciences,

University of the Philippines Los Baños, Laguna, Philippines

*Corresponding author: galias.deancarlo@gmail.com

Date Submitted: 07 September 2017

Date Accepted: 10 April 2018 ancestral domain of the Magbukún Aeta, who rely heavily on forest resources for food, medicine, clothing and shelter. The Magbukún Aeta reside in the Kanawan Negritos Reservation Area (KNRA) which, is part of the 10,000 ha ancestral domain of the Aeta that includes some portions of the Subic Bay Forest Reserve (south of Boton River) and a portion of the Bataan Natural Park (BNP) on the northwestern side of Mt. Natib in Morong, Bataan (Cruz, 2010). The BNP is 23,688 ha and is severely deforested (PTFCF, 2013).

The destruction of tropical forests results in the decline in biodiversity and disruption of ecosystem services (Diereck and Holscher, 2009). In the case of the Aeta (Magbukún tribe of Morong, Bataan), the present ancestral domain covering 10,000 ha that was once forested is now much reduced. With the forest degradation within their ancestral domain and reducing their sources of food, the Aeta have been marginalized and are living below the poverty line with a per capita income of 0.47 USD/day (Cruz, 2010).

This study conducted a vegetational analysis of the canopy tree species to determine the community structure of the remaining forest within the KNRA. The result of the study 
led to the inventory of the tree species present in the remaining forest of the KNRA. This information was used in determining the relative importance of each tree species and in obtaining estimates of tree population densities. These data were used in determining the successional stages of the forest. This study is also valuable for the conservation, rehabilitation and sustainability of the remnant native forest.

\section{MATERIALS AND METHODS}

Study Area. The study was carried out within the 188 ha KNRA in Sitio Kanawan, Barangay Binaritan in Morong, Bataan $\left(14^{\circ} 41^{\prime} \mathrm{N}\right.$ and $\left.120^{\circ} 16^{\prime} \mathrm{E}\right)$ (Fig. 1). Kanawan is on a ridge of Mt. Silangan located across the Bataan River from the Bataan Technological Park (Motin, et al., 2006). Bataan province lies in the entirety of the Bataan Peninsula, along the west coast of Luzon island facing the West Philippine Sea. It is $80 \mathrm{~km}$ west of Manila, $175 \mathrm{~km}$ by road to the north around Manila Bay and then through the rugged terrain of Bataan Province (Bustos, et al., 1997). Bataan has an average annual temperature of $26.1^{\circ} \mathrm{C}$, coolest during the months of November to December $\left(23.3^{\circ} \mathrm{C}\right)$ and warmest during April $\left(28.6^{\circ} \mathrm{C}\right)$. The average annual relative humidity is $78 \%$, ranging from $73 \%$ in May to $83 \%$ in July. The monthly rainfall varies from 0 (during the dry months of January to May) to $314.6 \mathrm{~mm}$ (during the rainy months of June to September), with a mean precipitation of $66.7 \mathrm{~mm}$. The rainfall pattern can be variable through the years depending on the onset and end of the monsoon rains (Bustos, et al., 1997).

Plant Inventory. The tree vegetation analysis was conducted within the two identified forests of the KNRA (Site 1/Hulo: 12.11 ha, Site 2/Buod: 15.14 ha). The PCQM of MuellerDombois and Ellenberg (1974) was used where five $200 \mathrm{~m}$ transects were laid across the 28 ha study site. Points were set at $10-\mathrm{m}$ intervals. Trees with $\geq 1 \mathrm{~cm}$ diameter at $1.3 \mathrm{~m}$ from the ground (DBH) were sampled. Common tree species were identified on site by Aeta members who were trained on taxonomy, and using a pictorial guide of Galang and coworkers (2012). For plants unknown to them, leafy branches, flowers, fruits (if available) and other plant parts containing important characters were photographed and were brought to the University of the Philippines Los BañosCollege of Forestry and Natural Resources (UPLB- CFNR) for identification. Taxonomic information of all plant samples were verified using the on-line database of Pelser and coworkers (2011). Trees encountered were categorized into three groups: seedling, sapling and adult based on girth classes (Omeja, et al., 2004). Shannon-Wiener's and Simpson's diversity indices were also computed. The distribution and conservation status of plant samples were based on The IUCN Red List of Threatened Species (20173 ) and the Department of Environment and Natural Resources Administrative Order (DAO) (2017-11).
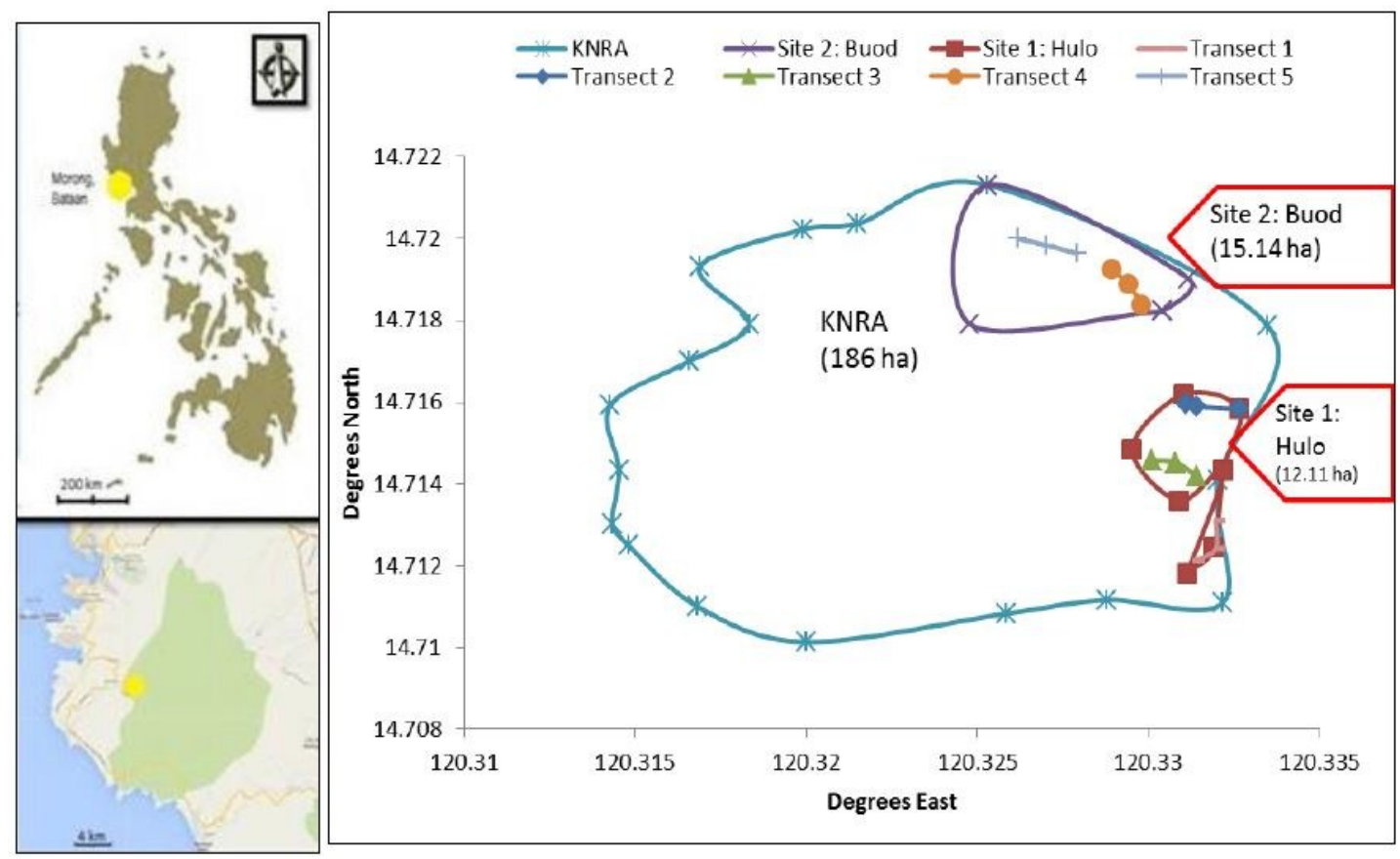

Figure 1. Map showing the location of the study area and the five transects as sampling sites for plant inventory under PCQM within the two forest patches of the KNRA. 


\section{RESULTS AND DISCUSSION}

Tree species composition of the two forests in the Kanawan Negritos Reservation Area

A total of 398 tree individuals were recorded from the five 200 $\mathrm{m}$ transects laid across the 28-ha KNRA. These were further classified into 76 species belonging to 61 genera of the 35 plant families (Fig. 2). Comparing the two forests, Site 1 is composed of 57 species from 46 genera of the 28 families. On the other hand, there were 46 species, 37 genera and 28 families recorded in Site 2 . All the trees encountered together with their distribution and conservation status are listed in Table 1.

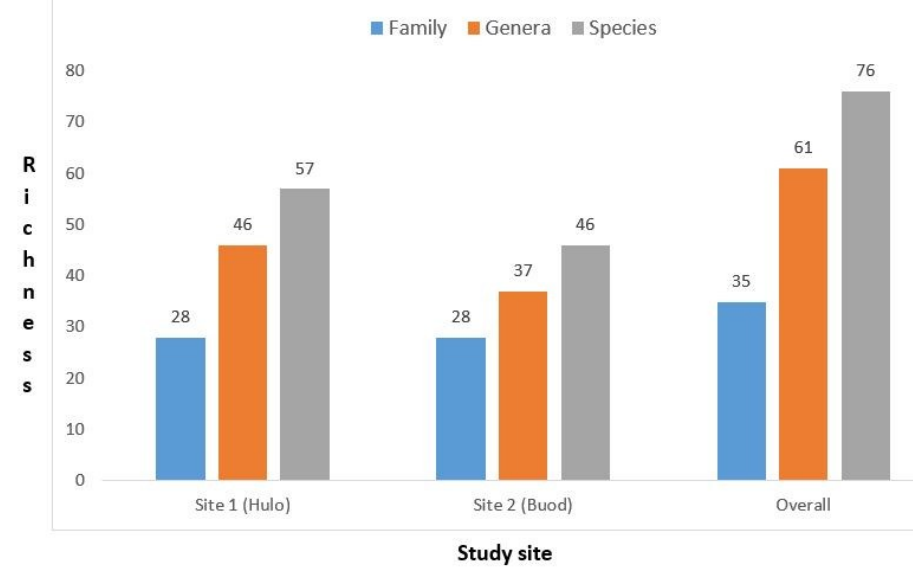

Figure 2. Species composition of the different sites

The most represented family was Moraceae, having eight species $(10.53 \%)$ composed of 71 individuals. Six out of the seven species of Moraceae are from the genus Ficus, which included $F$. benjamina L., $F$. nota (Blanco) Merr., $F$. odorata (Blanco) Merr., F. septica Burm. f., F. variegata Blume and $F$. subcordata Blume. The other two species representing this family were Artocarpus blancoi (Elmer) Merr. and $A$. ovatus Blanco. The result of the present study is comparable to the work of Arsenio et al., (2011) in Mt. Maculot in Batangas where the most represented family was Moraceae with seven species belonging to three genera. Also, the genus Ficus was the most represented genus with five species.

The study of Cottee-Jones and co-workers (2016), demonstrated the important role of Ficus trees in ecological succession. They found out that the density of saplings growing under Ficus trees, was twice as high as the density of saplings growing under other non-Ficus trees. Also, in terms of species richness, saplings under Ficus trees were also significantly higher. Their result indicated that Ficus trees are more effective restoration nuclei than other remnant tree types. Furthermore, Ficus is believed to be a very important genus of fleshy-fruited tree for a wide range of frugivores because in intact forests, its unusual asynchronous fruiting cycle, large crop sizes, and pan-tropical availability means that over 1,200 tropical birds and mammals have been recorded consuming its fruit (Shanahan, et al., 2001).

The second most represented families were the Dipterocarpaceae, Lauraceae and Sapindaceae all with six species $(7.89 \%)$ identified each. Next were the Fabaceae (6.58\%) and Anacardiaceae $(5.26 \%)$ having five and four species identified, respectively. The dipterocarp family was represented by Anisoptera thurifera (Blanco) Blume, Dipterocarpus gracilis Blume, D. grandiflorus Blanco, Hopea plagata (Blanco) Vidal, Shorea contorta Vidal, S. guiso (Blanco) Blume and S. polysperma (Blanco) Merr. The Sapindaceae was represented by Allophyllus cobbe (L.) Raeusch., Dimocarpus longan Lour. ssp. malesianus Leenh, Elattostachys sp., Ganophyllum falcatum Blume, Guioa koelreuteria (Blanco) Merr. and Mischocarpus sp. Although the Dipterocarpaceae only ranked second in terms of representative species; the most dominant species in each site based on importance percentage (IP) were dipterocarps. From these data we can summarize that the forest was once a dipterocarp forest.

Other families included were Rubiaceae and Euphorbiaceae both with three species (3.95\%) each, followed by the Apocynaceae, Cannabaceae, Clusiaceae, Phyllantaceae and Sterculiaceae each with two species identified. The remaining $25 \%$ was allotted to 19 families which all have one species recorded.

In terms of the total basal area cover, the Dipterocarpaceae dominated with $120,417 \mathrm{~cm}^{2}$ or $(32.28 \%)$ of the total basal area. Next was the Moraceae having a total basal area of $77,428 \mathrm{~cm}^{2}(20.76 \%)$. This was followed by the Sapindaceae at $32,626 \mathrm{~cm}^{2}(8.75 \%)$, and Burseraceae at $23,943 \mathrm{~cm}^{2}(6.42 \%)$.

According to the forest formations of the Philippines scheme by Fernando, et al., (2008) the study area can be classified as a tropical semi-evergreen rain forest. This is a type of forest formation where there is a yearly water stress of some duration or a very distinct strong dry season. Because of this pronounced dry season, the tropical semi evergreen rain forest, when cut, is more frequently replaced by grasses dominated by Imperata cylindrica (L) P. Beauv. and Saccharum spontaneum L. (Fernando, et al., 2008). In fact, almost three-fourths of the mountain where Site 2 (Buod) was located was already covered by different kinds of grasses, chiefly I. cylindrica.

This forest formation included both evergreen and deciduous trees in a mixture, but with a tendency towards 
Table 1. List of tree species $(\geq 1 \mathrm{~cm}$ at $1.3 \mathrm{~m}=\mathrm{DBH})$ observed using Point- Centered Quarter Method $(\mathrm{PCQM})$ in the forest of Kanawan Negritos Reservation Area (KNRA), Barangay Binaritan, Morong, Bataan, Philippines (27.25 ha). Elevation: 180 to 348 m.a.s.l.

\begin{tabular}{|c|c|c|c|}
\hline FAMILY / SPECIES & $\begin{array}{l}\text { COMMON } \\
\text { NAME }\end{array}$ & DISTRIBUTION & $\begin{array}{c}\text { SITE/S } \\
\text { LOCATED }\end{array}$ \\
\hline
\end{tabular}

\section{ANACARDIACEAE}

\section{Buchanania arborescens}

(Blume) Blume

Balinghasai

Koordersiodendron

pinnatum (Blanco) Merr.

Amugis

Mangifera altissima Blanco

Pahutan

Semecarpus paucinervia

Merr.

\section{ANNONACEAE}

Miliusa vidalii J. Sincl.

APOCYNACEAE

Alstonia scholaris (L.) R.

$\mathrm{Br}$.

\section{Tabernaemontana \\ pandacaqui Poir. in Lam.}

BOMBACACEAE

Bombax ceiba L.
Dita

Pandakaki

Ligas-ilanan

Takulau

Pandakaki

Malabulak
Tenasserim, Andaman Isls, Thailand, Indochina, Taiwan, across Malesia from Sumatra to New Britain, Solomon Isls and NE Australia. Throughout the Philippines, chiefly in lowland forests, along river banks, secondary forest 2 (Pelser, et al., 2011).

Philippines, Sulawesi, Moluccas, New Guinea, N. Luzon (Cagayan) to Palawan and Mindanao. Lowland forests, rarely up to $450 \mathrm{~m}$ elevation, on dryland or occasionally inundated areas (Pelser, et al., 2011).

\section{Other Threatened Species (DAO 2017-11)}

Common. Philippines, Sulawesi, Lesser Sunda Isls, Moluccas, New Guinea, S New Britain, Solomon Isls. LUZON: llocos Sur, Cagayan, Nueva Ecija, Zambales, Rizal, Laguna, Quezon, Camarines, MINDORO, PALAWAN, SIBUYAN. Chiefly in lowland primary forests whether inland or sometimes coastal, ascending to $400 \mathrm{~m}$ (Pelser, et al., 2011).

Borneo (Sabah), Philippines, PALAWAN: Mt Pulgar and Mt. Victoria. Lowland primary forests along stream bank (Pelser, et al., 2011).

Vulnerable (DAO 2017-11)

No available data.

Pakistan, Nepal, India, Bhutan, Bangladesh, Sri Lanka, China (Yunnan), Myanmar, Thailand, Cambodia, Laos, Vietnam, throughout Malesia, Solomon Isls and Australia (Queensland), Philippines. LUZON: (Cagayan) to PALAWAN and MINDANAO, in most or all islands and provinces. In primary and secondary forests at low and medium elevation (Pelser, et al., 2011).

LUZON: Bataan prov., Mt. Mariveles, Lamao River, S. China, Taiwan, Thailand, Java, Borneo (Sabah), Philippines, Sulawesi, Java, Lesser Sunda Isls, Moluccas, New Guinea, N and E parts of Australia, Micronesia. In the Philippines common in most islands and provinces (Pelser, et al., 2011).

India to S China, southward to Sumatra and Java, LUZON: Abra, Nueva Ecija, Bulacan, Bataan, Rizal, MINDORO, CAMIGUIN, MINDANAO. Scattered in secondary and primary forest at low and medium elevation (Pelser, et al., $1 \& 2$ 2011). 


\begin{tabular}{|c|c|c|c|}
\hline FAMILY / SPECIES & $\begin{array}{l}\text { COMMON } \\
\text { NAME }\end{array}$ & DISTRIBUTION & $\begin{array}{c}\text { SITE/S } \\
\text { LOCATED }\end{array}$ \\
\hline
\end{tabular}

\section{BURSERACEAE}

Canarium europhyllum

Perkins

Canarium luzonicum

(Blume) A. Gray

BYTTNERIACEAE

Kleinhovia hospita L. Tan-ag

CALOPHYLLACEAE

Calophyllum inophyllum L. Bitaog

\section{CANNABACEAE}

Celtis philippinensis Blanco Malaikmo

Trema orientalis (L.) Blume Anabiong

\section{CHRYSOBALANACEAE}

Maranthes corymbosa

Blume

Liusin

Garcinia sp.

Terminalia foetidissima

Griff.
Throughout the Philippines except Palawan. Rather common in lowland forests, ascending to $800 \mathrm{~m}$ (Pelser, et al., 2011).

$1 \& 2$

Endemic. LUZON, ALABAT, MINDORO, MASBATE, TICAO, BOHOL, MINDANAO (Pelser, et al., 2011).

Other Threatened Species (DAO 2017-11)

$1 \& 2$

Tropical Africa and Asia. Throughout the Philippines, at low and medium elevation, a characteristic tree of thickets, secondary growth forests, and deserted clearings (Pelser, et al., 2011).

Tropical E. Africa to India, SE Asia, Hainan, Taiwan, Ryukyu Isls, across Malesia to N. Australia, New Caledonia to Polynesia. Introduced to the Neotropics and $1 \& 2$ tropical W Africa (Pelser, et al., 2011).

Tropical Africa to Madagascar, India, Myanmar, SE China, Hongkong, Taiwan, Thailand, Indochina, throughout Malesia to NE Australia. N LUZON to MINDANAO and PALAWAN (Pelser, et al., 2011).

Tropical Africa, Sri Lanka, India, Myanmar, Thailand, Indochina, China, Hainan, Taiwan, S Japan, Sumatra, Peninsular Malaysia, Java, Lesser Sunda Isls, Borneo, Philippines, Sulawesi, Moluccas, New Guinea (including New Britain), Australia (Queensland), Solomon Isls, Marianas, Polynesia (Fiji; Tonga; Tahiti) (Pelser, et al., 2011).

S. Thailand, Sumatra, Peninsular Malaysia, Java, Borneo, Philippines, Sulawesi, Lesser Sunda Isls, Moluccas, New Guinea, New Britain, Admiralty Isls, Solomon Isls, Caroline Isls, Australia (Queensland, Northern Territory). N LUZON to PALAWAN and MINDANAO. Lowland and medium elevation forests (Pelser, et al., 2011).

Peninsular Malaysia (Selangor, Negeri Sembilan, Melaka), S- Burma (Taninthayi, Yangon), S-Thailand, Sumatra, Borneo, Philippines (Luzon, Mindoro, Panay, Mindanao) 2 (Pelser, et al., 2011). 


\begin{tabular}{|c|c|c|c|}
\hline FAMILY / SPECIES & $\begin{array}{l}\text { COMMON } \\
\text { NAME }\end{array}$ & DISTRIBUTION & $\begin{array}{c}\text { SITE/S } \\
\text { LOCATED }\end{array}$ \\
\hline
\end{tabular}

\section{DIPTEROCARPACEAE}

\begin{abstract}
Anisoptera thurifera
(Blanco) Blume
\end{abstract}

Dipterocarpus gracilis

Blume

Panau

Dipterocarpus grandiflorus

Blanco

Apitong

Hopea plagata (Blanco)

Vidal

Shorea contorta Vidal

Shorea guiso (Blanco)

Blume

Guijo

Bulong-eta

Blanco

ELAEOCARPACEAE

Elaeocarpus curanii Merr.
It is native to the Philippines, Indonesia and Papua New Guinea (Pelser, et al., 2011).

Vulnerable (IUCN 2017-3)

$1 \& 2$

The species is native to India, Bangladesh, Indochina, Borneo and the Philippines. Within Indochina the species is found in Myanmar, Thailand, Peninsular Malaysia and Singapore. (Pelser, et al., 2011).

Vulnerable (IUCN 2017-3, DAO 2017-11)

The species is native to India, Indochina, the Philippines, Indonesia and Sabah. It is found in India (Andaman Islands), Vietnam, Myanmar, Thailand, Peninsular Malaysia and Singapore (Pelser, et al., 2011).

Endangered (IUCN 2017-3), Vulnerable (DAO 2017-11)

Native: Malaysia (Sarawak); Philippines (IUCN) NE Borneo and the Philippines. LUZON: Cagayan, Nueva Vizcaya, Nueva Ecija, Pangasinan, Bulacan, Bataan, Rizal, Laguna, Saplungan Quezon, Camarines, Sorsogon, Mindoro, Tablas, Bohol, Mindanao, Basilan, Tawi Tawi (Pelser, et al., 2011). Vulnerable (DAO 2017-11)

Endemic to the Philippines. Babuyan Isls. (Calayan), LUZON (in most provinces), POLILLO, MINDORO, SIBUYAN, MARINDUQUE, MASBATE, NEGROS, LEYTE, SAMAR, MINDANAO: Zamboanga, Lanao, Agusan, BASILAN. Common in primary lowland forests from semiseasonal to everwet areas, in the former often semigregarious (Pelser, et al., 2011).

Critically endangered (IUCN 2017-3), Vulnerable (DAO 2017-11)

Peninsular and SE Thailand, S Vietnam, Peninsular Malaysia, Sumatra (Aceh, Tapanuli, Palembang), Borneo (mainly in E), Philippines. LUZON (widespread in most provinces), Mindoro, Masbate, Panay, Negros, Leyte, Samar, Mindanao, Basilan (Pelser, et al., 2011).

$1 \& 2$

Luzon: Batangas Province, Punta Santiago, in dry forest (Pelser, et al., 2011).

$1 \& 2$

Vulnerable (DAO 2017-11)

LUZON: Ilocos Norte (Pasuquin), Cagayan, Zambales, Bataan, Pampanga, Quezon, Camarines. Low elevation 2 forests (Pelser, et al., 2011) 


\begin{tabular}{|c|c|c|c|}
\hline FAMILY / SPECIES & $\begin{array}{l}\text { COMMON } \\
\text { NAME }\end{array}$ & DISTRIBUTION & $\begin{array}{l}\text { SITE/S } \\
\text { LOCATED }\end{array}$ \\
\hline \multicolumn{4}{|l|}{ EUPHORBIACEAE } \\
\hline Fahrenheitia sp. & - & - & 2 \\
\hline $\begin{array}{l}\text { Macaranga grandiflora } \\
\text { (Blanco) Merr. }\end{array}$ & Takip- asin & $\begin{array}{l}\text { Endemic. LUZON: La Union, Benguet, Cagayan, Nueva } \\
\text { Vizcaya, Pangasinan, Bataan, Rizal, Batangas, Laguna, } \\
\text { Quezon, MINDORO (Pelser, et al., 2011). } \\
\text { Vulnerable (DAO 2017-11) }\end{array}$ & $1 \& 2$ \\
\hline $\begin{array}{l}\text { Macaranga tanarius (L.) } \\
\text { Muell.-Arg. }\end{array}$ & Binunga & $\begin{array}{l}\text { Nicobar Isls, Andaman Isls, Thailand, S China, Taiwan, } \\
\text { Ryukyu Isls, Sumatra, Peninsular Malaysia, Java, Borneo, } \\
\text { Philippines, Sulawesi, Lesser Sunda Isls, Moluccas, New } \\
\text { Guinea, NE Australia, Melanesia, Micronesia (Pelser, et } \\
\text { al., 2011). }\end{array}$ & $1 \& 2$ \\
\hline \multicolumn{4}{|l|}{ FABACEAE } \\
\hline $\begin{array}{l}\text { Afzelia rhomboidea } \\
\text { (Blanco) Vidal }\end{array}$ & Tindalo & $\begin{array}{l}\text { Sumatra, Borneo (Sabah, Kalimantan), and the } \\
\text { Philippines. N Luzon to Palawan and Mindanao (Pelser, et } \\
\text { al., 2011). Vulnerable (IUCN 2017-3), Endangered (DAO } \\
\text { 2017-11) }\end{array}$ & 1 \\
\hline $\begin{array}{l}\text { Archidendron merrillii } \\
\text { (J.F.Macbr.) I.C. Nielsen }\end{array}$ & $\begin{array}{l}\text { Anagap- } \\
\text { bangin }\end{array}$ & Luzon: Benguet Prov., W of Baguio (Pelser, et al., 2011). & 1 \\
\hline Cynometrasp. & - & - & 1 \\
\hline Millettia merillii Perkins & "Malabai" Pam & $\begin{array}{l}\text { Endemic. LUZON: Cagayan to Bataan, Bulacan, Rizal, } \\
\text { Laguna (Mt Makiling), MINDORO, PALAWAN, NEGROS. } \\
\text { Lowland and medium elevation thickets and secondary } \\
\text { forests, also along riverbanks. Locally abundant (Pelser, et } \\
\text { al., 2011). Other Threatened Species (DAO 2017-11) }\end{array}$ & $1 \& 2$ \\
\hline $\begin{array}{l}\text { Parkia timoriana (DC.) } \\
\text { Merr. }\end{array}$ & Kupang & $\begin{array}{l}\text { NE India, Bangladesh, Burma, Thailand, Sumatra, } \\
\text { Peninsular Malaysia, Java, Lesser Sunda Isls (W } \\
\text { Sumbawa; Timor), Borneo, Philippines, Sulawesi, } \\
\text { Moluccas, New Guinea (W Irian). LUZON: La Union to } \\
\text { Laguna, PALAWAN (Pelser, et al., 2011). }\end{array}$ & 2 \\
\hline \multicolumn{4}{|l|}{ LAMIACEAE } \\
\hline Vitex turczaninowii Merr. & Lingo- lingo & $\begin{array}{l}\text { LUZON: llocos Sur, Pangasinan, Cagayan, Isabela, } \\
\text { Bataan, Rizal Laguna, Quezon, Camarines, Sorsogon, } \\
\text { MINDORO, TICAO, LEYTE, MINDANAO: Agusan, } \\
\text { Surigao, Lowland forests (Pelser, et al., 2011). }\end{array}$ & 2 \\
\hline \multicolumn{4}{|l|}{ LAURACEAE } \\
\hline $\begin{array}{l}\text { Alseodaphne longipes } \\
\text { Quisumb. \& Merr. }\end{array}$ & Babulo & $\begin{array}{l}\text { LUZON: Benguet (Tania), Isabela (Mt. Moises), Nueva } \\
\text { Vizcaya (Caraballo Mtns), Quirino (Mt Alzapan), Benguet, } \\
\text { MINDANAO (Agusan del Norte). Forests, sometimes } \\
\text { streams, } 970-1700 \mathrm{~m} \text { (Pelser, et al., 2011). }\end{array}$ & $1 \& 2$ \\
\hline
\end{tabular}




\begin{tabular}{|c|c|c|c|}
\hline FAMILY / SPECIES & $\begin{array}{l}\text { COMMON } \\
\text { NAME }\end{array}$ & DISTRIBUTION & $\begin{array}{c}\text { SITE/S } \\
\text { LOCATED }\end{array}$ \\
\hline $\begin{array}{l}\text { Cryptocarya acuminata } \\
\text { Merr. }\end{array}$ & Karaskas & $\begin{array}{l}\text { MINDORO: Mindoro Oriental prov. Mt. Halcon. LUZON: } \\
\text { Isabela, Pampanga, MINDORO: Mt. Halcon, type locality, } \\
\text { Bongabong River. In primary forests at low elevation, } \\
\text { ascending to } 500 \mathrm{~m} \text { (Pelser, et al., 2011). }\end{array}$ & $1 \& 2$ \\
\hline Cryptocarya sp. & - & - & 1 \\
\hline $\begin{array}{l}\text { Litsea grandis (Wall. ex } \\
\text { Nees) Hook.f. }\end{array}$ & Marang lapara & $\begin{array}{l}\text { Myanmar, Thailand, Sumatra, Peninsular Malaysia, } \\
\text { Singapore, Borneo, Philippines, LUZON: Isabela, Aurora, } \\
\text { Quezon, Camarines, SAMAR, MINDANAO: Zamboanga, } \\
\text { Misamis, Lanao. Primary forest at low and medium } \\
\text { elevation (Pelser, et al., 2011). }\end{array}$ & $1 \& 2$ \\
\hline $\begin{array}{l}\text { Litsea glutinosa (Lour.) } \\
\text { C.B. Rob. }\end{array}$ & Sablot & $\begin{array}{l}\text { India to S China through Malesia to tropical Australia, } \\
\text { Throughout the Philippines, in secondary and primary } \\
\text { forests at low and medium elevation (Pelser, et al., 2011). }\end{array}$ & $1 \& 2$ \\
\hline Litsea sp. & - & - & 1 \\
\hline \multicolumn{4}{|l|}{ LECYTHIDACEAE } \\
\hline $\begin{array}{l}\text { Barringtonia racemosa (L.) } \\
\text { Blume ex DC. }\end{array}$ & Putat & $\begin{array}{l}\text { E \& S Africa (Kenya; Tanzania, incl. Zanzibar; } \\
\text { Mozambique; Natal), Mafia Is, Madagascar, Comoros, } \\
\text { Seychelles, India (Malabar and Coromandel coasts), Sri } \\
\text { Lanka, Bangladesh (Sundarbans), Andaman Isls, Nicobar } \\
\text { Isls, Myanmar, S Thailand, Laos, Hainan, Taiwan, Ryukyu } \\
\text { Isls (Iriomote), throughout Malesia, Bismarck Archipelago, } \\
\text { Vanuatu, Marianas (Guam), Caroline Isls. (Palau, Yap, } \\
\text { Truk, Ponape), NE Australia (Queensland), New } \\
\text { Caledonia, Fiji, Samoa (Pelser, et al., 2011). }\end{array}$ & 1 \\
\hline \multicolumn{4}{|l|}{ LEEACEAE } \\
\hline Leea philippinensis Merr. & Kaliantan & $\begin{array}{l}\text { Endemic. Philippines: BATAN, LUZON: Apayao, Benguet, } \\
\text { Pangasinan, Cagayan, Isabela, Nueva Ecija, Aurora, } \\
\text { Zambales, Rizal, Quezon, Laguna (Mt Makiling), } \\
\text { MINDORO: Mindoro Oriental, Mindoro Occidental, } \\
\text { MINDANAO: Zamboanga del Norte, Davao, Surigao } \\
\text { (Pelser, et al., 2011). }\end{array}$ & $1 \& 2$ \\
\hline \multicolumn{4}{|l|}{ LYTHRACEAE } \\
\hline $\begin{array}{l}\text { Lagerstroemia speciosa } \\
\text { (L.) Pers. }\end{array}$ & Banaba & $\begin{array}{l}\text { Borneo, Cambodia, China (Yunnan), India, Java, Laos, } \\
\text { Lesser Sunda Isls, Moluccas, Myanmar, Peninsular } \\
\text { Malaysia, Sulawesi, Sumatra, Thailand, Vietnam, } \\
\text { Philippines. LUZON, MINDORO, PALAWAN, LEYTE, } \\
\text { MINDANAO, SAMAR. Mostly in secondary forests at low } \\
\text { and medium elevation (Pelser, et al., 2011). }\end{array}$ & 2 \\
\hline \multicolumn{4}{|l|}{ MAGNOLIACEAE } \\
\hline Elmerifollia sp. & - & - & 1 \\
\hline
\end{tabular}




\begin{tabular}{|c|c|c|c|}
\hline FAMILY / SPECIES & $\begin{array}{l}\text { COMMON } \\
\text { NAME }\end{array}$ & DISTRIBUTION & $\begin{array}{c}\text { SITE/S } \\
\text { LOCATED }\end{array}$ \\
\hline
\end{tabular}

\section{MALVACEAE}

Pterospermum celebicum Miq.

Bayokbayokan

\section{MORACEAE}

Artocarpus blancoi (Elmer) Merr.

Antipolo

Artocarpus ovatus Blanco

Anubing

Ficus benjamina L.

Ficus nota (Blanco) Merr. Tibig

Ficus odorata (Blanco)

Merr.

Pakiling

Ficus septica Burm.f.

Hauili

Ficus subcordata Blume
Taiwan, Sulawesi and the Moluccas. All over the Philippines especially in Luzon. Primary and secondary forests particularly in lower forested regions, in rocky woods, including river banks, open areas

Endemic. BATAN, LUZON, MINDORO, PALAWAN, NEGROS, CEBU, MINDANAO. Thickets and forests at low and medium elevation (Pelser, et al., 2011). Vulnerable (IUCN 2017-3)

LUZON: Abra (Manabo), Kalinga (Lubuagan), Benguet, Isabela (San Mariano), Nueva Vizcaya (near Dupax), Nueva Ecija, Zambales (Mt. Pinatubo), Bataan (Lamao), Pampanga, Bulacan (Angat), Rizal (Mt. Angilog, Montalban, Antipolo, Bosoboso), Laguna (Los Baños, Mt. Makiling, Sta. Maria, Mabitac), Quezon (Laguimanoc, Padre Burgos, Lucban), Camarines Sur (Mt. Bagacay, Mt. Isarog), Sorsogon (Mt. Bulusan), CATANDUANES, MINDORO: Mindoro Oriental (Mansalay, Manaul and Mt. Yagaw), PALAWAN (Irawan), BALABAC (Cape Melville), MARINDUQUE, SIBUYAN (Mt. Giting-giting), MASBATE, TICAO, PANAY: Iloilo, NEGROS: Negros Occidental (Danao, Cadiz), Negros Oriental (Cuernos Mtns), CEBU. In forest $760 \mathrm{~m}$, often common (Pelser, et al., 2011).

India, S China, Myanmar, Thailand, Indochina to Australia (Arnhem Land and Queensland). Throughout Malesia. Philippines (Batan, Luzon, Mindoro), Forests up to $1300 \mathrm{~m}$. Often in secondary growth or planted (Pelser, et al., 2011).

N Borneo, Philippines. Lowland montane forest, often along stream, up to 1300 m (Pelser, et al., 2011).

BATANES, LUZON, PANAY, NEGROS, BOHOL, LEYTE, SAMAR, MINDANAO. Lowland forests, often in secondary, near streams, and on rocks (Pelser, et al., 2011).

NE India to S China, Taiwan, throughout Malesia to Australia (Queensland) to Vanuatu. Lowland and montane forests or secondary growth, often near rivers, up to 1800 m (Pelser, et al.,2 011).

Thailand, Indochina, Sumatra, Peninsular Malaysia, Java, Borneo, Philippines, Sulawesi, Lesser Sunda Isls (Bali, Flores, Sumbawa, Timor), New Guinea, New Britain. Forests, up to $1000 \mathrm{~m}$ (Pelser, et al.,2011). 


\begin{tabular}{|c|c|c|c|}
\hline FAMILY / SPECIES & $\begin{array}{l}\text { COMMON } \\
\text { NAME }\end{array}$ & DISTRIBUTION & $\begin{array}{c}\text { SITE/S } \\
\text { LOCATED }\end{array}$ \\
\hline Ficus variegata Blume & $\begin{array}{l}\text { Tangisang- } \\
\text { bayawak }\end{array}$ & $\begin{array}{l}\text { NE India to Myanmar, S China, Taiwan, Ryukyu Isls, } \\
\text { Indochina, S Andaman Isls, and to Thailand, Malesia, the } \\
\text { Solomon Isls, and Australia (Queensland). In Malesia } \\
\text { throughout the region. Forest and secondary growth up to } \\
1200 \mathrm{~m} \text {; often villages and gardens (Pelser, et al., 2011). }\end{array}$ & 1 \\
\hline \multicolumn{4}{|l|}{ MYRISTICACEAE } \\
\hline $\begin{array}{l}\text { Myristica philippinensis } \\
\text { Lam. }\end{array}$ & Duguan & $\begin{array}{l}\text { Endemic to the Philippines. Throughout the Philippines } \\
\text { (except Palawan). Primary lowland forests up to c. } 400 \mathrm{~m} \\
\text { (Pelser, et al., 2011) Other Threatened Species. (DAO } \\
\text { 2017-11) }\end{array}$ & $1 \& 2$ \\
\hline \multicolumn{4}{|l|}{ MYRTACEAE } \\
\hline Syzygium sp. & Malaruhat pula & - & $1 \& 2$ \\
\hline \multicolumn{4}{|l|}{ PHYLLANTHACEAE } \\
\hline Bischofia javanica Blume & Tuai & $\begin{array}{l}\text { Australia, Bangladesh, Borneo, China, Cook Isls, Fiji, India, } \\
\text { Japan, Java, Laos. Lesser Sunda Isls, Malay Peninsula, } \\
\text { Moluccas, Nepal, New Caledonia, New Guinea, Solomon } \\
\text { Isls., Sulawesi, Sumatra, Taiwan, Thailand, Tonga, } \\
\text { Vanuatu, Vietnam, Philippines. Throughout the Philippines. } \\
\text { Pioneer species up to } 1500 \text { (-2350) m (Pelser, et al., } \\
\text { 2011). }\end{array}$ & 2 \\
\hline $\begin{array}{l}\text { Cleistanthus angustifolius } \\
\text { Merr. }\end{array}$ & Aniatan kitid & $\begin{array}{l}\text { Endemic to the Philippines. LUZON: Cagayan, BUCAS } \\
\text { GRANDE. On forested ridges and slopes at low elevation } \\
\text { (Pelser, et al., 2011). }\end{array}$ & 1 \\
\hline \multicolumn{4}{|l|}{ POLYGALACEAE } \\
\hline $\begin{array}{l}\text { Xanthophyllum flavescens } \\
\text { Roxb. }\end{array}$ & - & $\begin{array}{l}\text { Throughout continental SE Asia, Sumatra, Simalur, Banka, } \\
\text { Peninsular Malaysia, Borneo, Philippines, LUZON: } \\
\text { Benguet, Cagayan, Nueva Ecija, Zambales, Bataan, Rizal, } \\
\text { Laguna, Albay, Sorsogon, MINDORO, BUSUANGA, } \\
\text { CULION, PALAWAN, MASBATE, GUIMARAS, NEGROS, } \\
\text { MINDANAO: Zamboanga, Low and medium elevation } \\
\text { forests (Pelser, et al., 2011). }\end{array}$ & $1 \& 2$ \\
\hline \multicolumn{4}{|l|}{ RUBIACEAE } \\
\hline $\begin{array}{l}\text { Kanapia monstrosa (A. } \\
\text { Rich.) Arriola \& Alejandro }\end{array}$ & $\begin{array}{l}\text { Tadiang- } \\
\text { anuang }\end{array}$ & $\begin{array}{l}\text { Sulawesi, Philippines. LUZON: Aurora, Camarines Sur, } \\
\text { llocos Norte, llocos Sur, Abra, La Union, Apayao, Mt. } \\
\text { Province, Benguet, Cagayan, Isabela, Nueva Vizcaya, } \\
\text { Pangasinan, Zambales, Bataan, Pampanga, Bulacan, } \\
\text { Rizal, Laguna, Cavite, Quezon, LEYTE, MINDORO, } \\
\text { NEGROS. Low and medium elevation secondary forests, } \\
\text { often common (Pelser, et al., 2011). }\end{array}$ & $1 \& 2$ \\
\hline $\begin{array}{l}\text { Morinda citrifolia L. var. } \\
\text { bracteata (Roxb.) Kurz }\end{array}$ & Nino & $\begin{array}{l}\text { Malesia (widespread). Throughout the Philippines in most } \\
\text { islands and provinces. Lowland thickets and secondary } \\
\text { forests (Pelser, et al., 2011). }\end{array}$ & 1 \\
\hline
\end{tabular}




\begin{tabular}{cccc}
\hline FAMILY / SPECIES & $\begin{array}{c}\text { COMMON } \\
\text { NAME }\end{array}$ & DISTRIBUTION & SITE/S \\
LOCATED
\end{tabular}

\begin{tabular}{lll}
\hline $\begin{array}{l}\text { Wendlandia philippinensis } \\
\text { Cowan }\end{array}$ & Mamboi & Endemic to the Philippines. LUZON: Benguet Province, \\
& Daklan to Kabayan (Pelser, et al., 2011).
\end{tabular}

\section{SALICACEAE}

Casearia fuliginosa

(Blanco) Blanco

Talitan

\section{SAPINDACEAE}

Allophyllus cobbe (L.)

Raeusch.

Dimocarpus longan Lour. ssp. malesianus Leenh.

Elattostachys sp.

Ganophyllum falcatum

Blume

Guioa koelreuteria (Blanco) Merr.

Mischocarpus sp.

\section{SAPOTACEAE}

Palaquium philippense

(Perr.) C.B. Rob.

Malak-malak

Maniknik
LUZON, MINDORO, TICAO, MASBATE, SIBUYAN, SAMAR, DINAGAT, MINDANAO, TAWI-TAWI. Rather common in thickets and secondary forests below $250 \mathrm{~m}$, also near the sea, often on sandy or gravelly riverbanks and along creeks (Pelser, et al., 2011).

S. America, S. Africa, Madagascar, Myanmar, Indochina through Peninsular Malaysia to New Guinea. Widespread in the Philippines in most islands and provinces (Pelser, et al., 2011).

Myanmar, Laos, Cambodia, S. Vietnam, Sumatra, Peninsular Malaysia, Borneo, Sulawesi, Moluccas, Alupag Philippines LUZON: Bataan, Pampanga. Lowland forests (Pelser, et al., 2011). Near Threatened (IUCN 2017-3)

Andaman Isls, Nicobar Isls, across Malesia to Australia (Western,Australia Northern Territory and Queensland), Philippines (Pelser, et al., 2011).

Borneo, Throughout the Philippines in most islands and provinces, Low and medium elevation secondary growth forests, common (Pelser, et al., 2011).

Endemic to the Philippines. LUZON: Isabela, Bataan, Tarlac, Bulacan, Rizal, Batangas, Quezon, Camarines Sur, Albay, Sorsogon, MINDORO: Mindoro Occidental (Mt. Calavite), PANAY: Capiz, NEGROS, LEYTE (Ormoc, Lake Danao), MINDANAO: Davao (Mt. Apo). Common in lowland and medium elevation primary forests (Pelser, et al., 2011). Vulnerable (DAO 2017-11)

Endemic to the Philippines. LUZON: Bataan, Laguna, Quezon, Camarines Sur, Sorsogon, MINDORO. Common in primary forests and dry hill forests at low and medium $1 \& 2$
Palaquium tenuipetiolatum Merr. 


\begin{tabular}{|c|c|c|c|}
\hline FAMILY / SPECIES & $\begin{array}{l}\text { COMMON } \\
\text { NAME }\end{array}$ & DISTRIBUTION & $\begin{array}{c}\text { SITE/S } \\
\text { LOCATED }\end{array}$ \\
\hline \multicolumn{4}{|l|}{ SPARMANNIACEAE } \\
\hline Grewia multiflora Juss. & Danglin & $\begin{array}{l}\text { Peninsular Malaysia to New Guinea, LUZON: Cagayan to } \\
\text { Quezon, MINDORO, PALAWAN, GUIMARAS, CAMIGUIN, } \\
\text { MINDANAO. Common in thickets and secondary forests } \\
\text { (Pelser, et al., 2011). }\end{array}$ & 1 \\
\hline \multicolumn{4}{|l|}{ STEMONURACEAE } \\
\hline $\begin{array}{l}\text { Gomphandra luzoniensis } \\
\text { Merr.subsp. Iuzoniensis }\end{array}$ & Mabunot & $\begin{array}{l}\text { Endemic to the Philippines. LUZON: llocos Norte, llocos } \\
\text { Sur, La Union, Cagayan, Nueva Ecija, Bataan, Rizal, } \\
\text { Laguna, Cavite, Batangas, MINDORO, MARINDUQUE, } \\
\text { PALAWAN. Common in primary forests under partial } \\
\text { shade or along forest edges at low elevation, rarely to } 850 \\
\text { m (Pelser, et al., 2011). }\end{array}$ & 2 \\
\hline Gomphandra sp. & Balinghasai & - & 2 \\
\hline \multicolumn{4}{|l|}{ STERCULIACEAE } \\
\hline Heritiera sy/vatica Vidal & Dungon & $\begin{array}{l}\text { Sulawesi. Philippines LUZON (Cagayan to Albay), } \\
\text { MINDORO, MASBATE, PANAY, SAMAR. In dry primary } \\
\text { forests at low and medium elevations (Pelser, et al., 2011). }\end{array}$ & $1 \& 2$ \\
\hline $\begin{array}{l}\text { Pterocymbium tinctorium } \\
\text { (Blanco) Merr. }\end{array}$ & Taluto & $\begin{array}{l}\text { Myanmar, Sumatra, Peninsular Malaysia, Java, Lesser } \\
\text { Sunda Isls, Borneo (Sabah), Sulawesi, Philippines. } \\
\text { BABUYAN ISLS, LUZON (widespread from at least } \\
\text { Cagayan to Camarines Sur), PALAWAN, CAMIGUIN, } \\
\text { MINDANAO. In secondary or thin primary forests at low } \\
\text { and medium elevations (Pelser, et al., 2011). }\end{array}$ & 1 \\
\hline
\end{tabular}


gregariousness (Whitmore, 1990; Fernando, et al., 2008). Examples of evergreen trees recorded in the area included: Anisoptera thurifera (Blanco) Blume, Cleistanthus angustifolius Merr., Ficus variegata Blume, Hopea plagata (Blanco) Vidal, Kleinhovia hospita L., Koordersiodendron pinnatum (Blanco) Merr., Macaranga grandiflora (Blanco) Merr., Mangifera altissima Blanco, Palaquium philippinense (Perr.) C.B. Rob., Shorea contorta Vidal, S. guiso (Blanco) Blume and Trema orientalis (L.) Blume. Correspondingly, examples of deciduous and semi- deciduous trees included: Afzelia rhomboidea (Blanco) Vidal, Bischofia javanica Blume, Bombax ceiba L., Lagerstroemia speciosa (L.) Pers. and Parkia timoriana (DC.) Merr. Most of these evergreen and deciduous/ semi- deciduous tree species were also mentioned in the book of Fernando, et al., (2008). The lauanapitong type of dipterocarp forest described by Whitford (1911) and the apitong-lauan association referred to by (Fernando, et al., 2008) belonged to this forest formation.

\section{Categorization of the tree species according to diameter at $1.3 \mathrm{~m}(\mathrm{DBH})$}

To identify the regeneration status of the forest, the different tree species encountered were categorized according to different diameter classes (Fig. 3). Analysis of data from PCQM showed that $34.45 \%$ of the individuals in Site 1 (Hulo) belonged to the seedlings class and $30.25 \%$ were saplings. Furthermore, $22.69 \%$ were pole trees and only about $12.61 \%$ were standard trees. Some of these pole trees and standard trees could be the possible mother trees of these seedlings and saplings. It is noteworthy that as the diameter class increases, its abundance decreases. This might mean that not all of the individuals were able to proceed to the next class. There were three large trees in this area having a DBH of $\geq 100 \mathrm{~cm}$ : Mischocarpus sp. (kamingi) (DBH: $143.2 \mathrm{~cm}$ ), Ficus subcordata Blume (payapa) (DBH: $137.8 \mathrm{~cm})$, and Ganophyllum falcatum (matang-ulang) (DBH: $101.5 \mathrm{~cm})$. Next bigger trees were Macaranga tanarius (L.) Muell.-Arg. (binunga) $(90.1 \mathrm{~cm})$ and Dimocarpus longan ssp. malesianus (alupag lalaki) $(84.0 \mathrm{~cm})$. These trees were the possible remnants of the original primary forest in the area.

The top two seedling species in Site 1 were Shorea contorta Vidal (white lauan) (14.6\%) and Canarium europhyllum (kila) (8.5\%) both of which are climax species (Table 2). The presence of these species suggested that the conditions in this remaining forest were favorable for growth and development of climax tree species that can pave the way for the regeneration of the former forest stand. It was also interesting to note that white lauan is an endemic species and is considered as a critically endangered species (per IUCN Red List 2017-3). As shown in Table 3, among the top 10 sapling species in this site were four endemic and/or

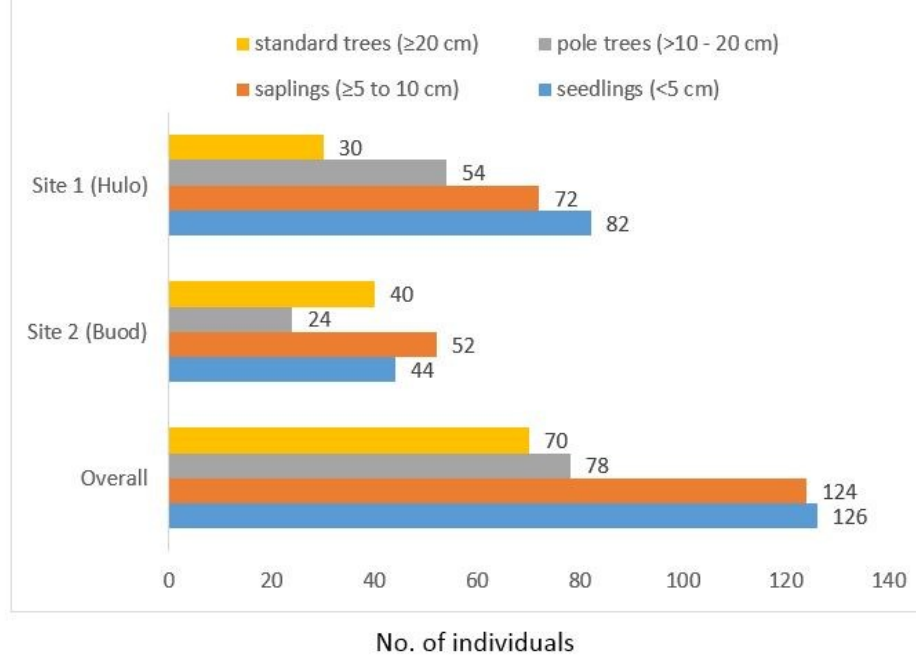

Figure 3. Categorization of tree species encountered according to diameter classes

threatened species which included Leea philippinensis Merr., Shorea contorta Vidal, Diospyros pilosanthera Blanco and Artocarpus blancoi (Elmer) Merr. The pole and standard trees were dominated by Macaranga tanarius (L.) Muell.-Arg. $(25.9 \%)$ and Shorea contorta Vidal (20\%), respectively (Tables $3 \& 4$ ).

On the other hand, $33.3 \%$ of the seedlings in the Site 2 (Buod) were all considered as threatened species. They were: Hopea plagata (Blanco) Vidal (yakal), Anisoptera thurifera (Blanco) Blume (palosapis), Diospyros pilosanthera Blanco (bulongeta), Shorea guiso (Blanco) Blume (guiso) and Dimocarpus longan ssp. malesianus (alupag lalaki), Macaranga grandiflora (Blanco) Merr. (takip-asin), Myristica philippinensis Lam. (duguan), and Shorea contorta Vidal (white lauan). Furthermore the top sapling was the endemic Leea philippinensis Merr. (14.7\%) (Table 3). Ranked $5^{\text {th }}$ was Diospyros pilosanthera Blanco (bulongeta) which is considered as an endangered species under DAO No. 201711. There were also three threatened pole trees in this site. Finally, the largest adult tree species in this site were Alseodaphne longipes (malabunga) $(150.2 \mathrm{~cm})$, Myristica philippinensis Lam. (tambaaw) $(118.7 \mathrm{~cm})$, Macaranga tanarius (L.) Muell.-Arg. (Binunga) $(105.7 \mathrm{~cm})$ and Canarium luzonicum (Blume) A. Gray (bulaig) $(101.9 \mathrm{~cm})$. Sixty per cent $(60 \%)$ of the top 10 standard trees $(\mathrm{DBH} \geq 20 \mathrm{~cm})$ in this site were considered threatened species (Table 5).

Considering that there were only two transects laid here, it is worth mentioning that there were four species of adult dipterocarp species recorded. The average DBH of adult trees in this area was $61.2 \mathrm{~cm}$, which was close to the mean DBH for Hulo at $66.7 \mathrm{~cm}$, where three transects were laid. This can strongly suggest that, indeed, the area was 
Philippine Journal of Systematic Biology | Galias D.C. and V.C. Cuevas: The Regenerating Forest of Magbukún Aeta

Table 2. Undergrowth (seedlings; $\mathrm{DBH} \leq 5 \mathrm{~cm}$ ) encountered within the forest of KNRA.

\begin{tabular}{|c|c|c|c|c|c|}
\hline \multicolumn{3}{|c|}{ SITE 1 (HULO) } & \multicolumn{3}{|c|}{ SITE 2 (BUOD) } \\
\hline Rank & Species/ Family & $(\%)$ & Rank & Species/ Family & $(\%)$ \\
\hline 1 & ${ }^{* * S h o r e a ~ c o n t o r t a ~ V i d a l ~(D i p t e r o c a r p a c e a e) ~}$ & 14.6 & 1 & $\begin{array}{l}\text { *Hopea plagata (Blanco) Vidal } \\
\text { (Dipterocarpaceae) }\end{array}$ & 18.2 \\
\hline 2 & $\begin{array}{l}\text { Canarium europhyllum Perkins } \\
\text { (Burseraceae) }\end{array}$ & 8.5 & 2 & $\begin{array}{l}\text { **Anisoptera thurifera (Blanco) Blume } \\
\text { (Dipterocarpaceae) }\end{array}$ & 9.1 \\
\hline 3 & $\begin{array}{l}\text { Cleistanthus angustifolius Merr. } \\
\text { (Phyllantaceae) }\end{array}$ & 7.3 & & $\begin{array}{l}{ }^{* * D} \text { Diospyros pilosanthera Blanco } \\
\text { (Ebenaceae) }\end{array}$ & 9.1 \\
\hline \multirow[t]{2}{*}{4} & $\begin{array}{l}{ }^{* *} \text { Artocarpus blancoi (Elmer) Merr. } \\
\text { (Moraceae) }\end{array}$ & 6.1 & 3 & Ficus septica Burm. f. (Moraceae) & 6.8 \\
\hline & $\begin{array}{l}{ }^{* *} \text { Semecarpus paucinervia Merr. } \\
\text { (Anacardiaceae) }\end{array}$ & 6.1 & 4 & Artocarpus ovatus Blanco (Moraceae) & 4.5 \\
\hline 5 & Grewia multiflora Juss. (Sparmanniaceae) & 4.9 & & Ficus nota (Blanco) Merr. (Moraceae) & 4.5 \\
\hline \multirow[t]{3}{*}{6} & Pterospermum celebicum Miq. (Malvaceae) & 3.7 & & Gomphandra sp. (Stemonuraceae) & 4.5 \\
\hline & $\begin{array}{l}\text { **Canarium luzonicum (Blume) A. Gray } \\
\text { (Burseraceae) }\end{array}$ & 3.7 & & $\begin{array}{l}\text { Palaquium tenuipetiolatum Merr. } \\
\text { (Sapotaceae) }\end{array}$ & 4.5 \\
\hline & Syzygium sp. (Myrtaceae) & 3.7 & & $\begin{array}{l}\text { **Shorea guiso (Blanco) Blume } \\
\text { (Dipterocarpaceae) }\end{array}$ & 4.5 \\
\hline \multirow[t]{6}{*}{7} & Heritiera sy/vatica Vidal (Sterculiaceae) & 2.4 & 5 & $\begin{array}{l}\text { Calophyllum inophyllum L. } \\
\text { (Calophyllaceae) }\end{array}$ & 2.3 \\
\hline & $\begin{array}{l}\text { Xanthophyllum flavescens Roxb. } \\
\text { (Polygalaceae) }\end{array}$ & 2.4 & & $\begin{array}{l}\text { Canarium europhyllum Perkins } \\
\text { (Burseraceae) }\end{array}$ & 2.3 \\
\hline & $\begin{array}{l}\text { Palaquium tenuipetiolatum Merr. } \\
\text { (Sapotaceae) }\end{array}$ & 2.4 & & $\begin{array}{l}\text { Cratoxylum arboreum Elmer } \\
\text { (Clusiaceae) }\end{array}$ & 2.3 \\
\hline & $\begin{array}{l}\text { Guioa koelreuteria (Blanco) Merr. } \\
\text { (Sapindaceae) }\end{array}$ & 2.4 & & $\begin{array}{l}\text { Cryptocarya acuminata Merr. } \\
\text { (Lauraceae) }\end{array}$ & 2.3 \\
\hline & Ficus odorata (Blanco) Merr. (Moraceae) & 2.4 & & $\begin{array}{l}\text { **Dimocarpus longan ssp. malesianus } \\
\text { Leenh.(Sapindaceae) }\end{array}$ & 2.3 \\
\hline & $\begin{array}{l}\text { Palaquium philippense (Perr.) C.B. Rob. } \\
\text { (Sapotaceae) }\end{array}$ & 2.4 & & $\begin{array}{l}\text { Elaeocarpus curanii Merr. } \\
\text { (Elaeocarpaceae) }\end{array}$ & 2.3 \\
\hline \multirow[t]{4}{*}{8} & $\begin{array}{l}\text { A/seodaphne longipes Quisumb. \& Merr. } \\
\text { (Lauraceae) }\end{array}$ & 1.2 & & $\begin{array}{l}\text { Guioa koelreuteria (Blanco) Merr. } \\
\text { (Sapindaceae) }\end{array}$ & 2.3 \\
\hline & $\begin{array}{l}{ }^{* *} \text { Anisoptera thurifera (Blanco) Blume } \\
\text { (Dipterocarpaceae) } 1.2\end{array}$ & 1.2 & & $\begin{array}{l}\text { Heritiera sy/vatica Vidal } \\
\text { (Sterculiaceae) }\end{array}$ & 2.3 \\
\hline & $\begin{array}{l}\text { Archidendron merrillii (J.F. Macbr.) I.C. } \\
\text { Nielsen (Fabaceae) }\end{array}$ & 1.2 & & $\begin{array}{l}\text { Kanapia monstrosa (A.Rich.) Arriola \& } \\
\text { Alejandro(Rubiaceae) }\end{array}$ & 2.3 \\
\hline & Celtis philippinensis Blanco (Cannabaceae) & 1.2 & & $\begin{array}{l}\text { ** Macaranga grandiflora (Blanco) } \\
\text { Merr. (Euphorbiaceae) }\end{array}$ & 2.3 \\
\hline
\end{tabular}


Philippine Journal of Systematic Biology | Galias D.C. and V.C. Cuevas: The Regenerating Forest of Magbukún Aeta

\begin{tabular}{|c|c|c|c|c|c|}
\hline \multicolumn{3}{|c|}{ SITE 1 (HULO) } & \multicolumn{3}{|c|}{ SITE 2 (BUOD) } \\
\hline Rank & Species/ Family & $(\%)$ & Rank & Species/ Family & $(\%)$ \\
\hline & Cryptocarya sp. (Lauraceae) & 1.2 & & $\begin{array}{l}\text { Macaranga tanarius (L.) Muell.-Arg. } \\
\text { (Euphorbiaceae) }\end{array}$ & 2.3 \\
\hline & Cynometra sp. (Fabaceae) & 1.2 & & $\begin{array}{l}\text { **Myristica philippinensis Lam. } \\
\text { (Myristicaceae) }\end{array}$ & 2.3 \\
\hline & $\begin{array}{l}\text { Diospyros pilosanthera Blanco } \\
\text { (Ebenaceae) }\end{array}$ & 1.2 & & $\begin{array}{l}\text { Pterospermum celebicum Miq. } \\
\text { (Malvaceae) }\end{array}$ & 2.3 \\
\hline & Elmerifollia sp. (Magnoliaceae) & 1.2 & & $\begin{array}{l}\text { **Shorea contorta Vidal } \\
\text { (Dipterocarpaceae) }\end{array}$ & 2.3 \\
\hline & Garcinia sp. (Clusiaceae) & 1.2 & & $\begin{array}{l}\text { Xanthophyllum flavescens Roxb. } \\
\text { (Polygalaceae) }\end{array}$ & 2.3 \\
\hline & $\begin{array}{l}\text { Litsea glutinosa (Lour.) C.B. Rob. } \\
\text { (Lauraceae) }\end{array}$ & 1.2 & & & \\
\hline & $\begin{array}{l}\text { **Macaranga grandiflora (Blanco) Merr. } \\
\text { (Euphorbiaceae) }\end{array}$ & 1.2 & & & \\
\hline & $\begin{array}{l}{ }^{* *} \text { Mangifera altissima Blanco } \\
\text { (Anacardiaceae) }\end{array}$ & 1.2 & & & \\
\hline & $\begin{array}{l}\text { Shorea guiso (Blanco) Blume } \\
\text { (Dipterocarpaceae) }\end{array}$ & 1.2 & & & \\
\hline
\end{tabular}


Philippine Journal of Systematic Biology | Galias D.C. and V.C. Cuevas: The Regenerating Forest of Magbukún Aeta

Table 3. Intermediate layer (saplings; $\mathrm{DBH} \geq 5-10 \mathrm{~cm}$ ) encountered within the forest of KNRA.

\begin{tabular}{|c|c|c|c|c|c|}
\hline \multicolumn{3}{|c|}{ SITE 1 (HULO) } & \multicolumn{3}{|c|}{ SITE 2 (BUOD) } \\
\hline Rank & Species/ Family & $(\%)$ & Rank & Species/ Family & $(\%)$ \\
\hline 1 & Ficus nota (Blanco) Merr. (Moraceae) & 15.3 & 1 & Leea philippinensis Merr. (Leeaceae) & 19.2 \\
\hline 2 & Leea philippinensis Merr. (Leeaceae) & 13.9 & 2 & Gomphandra sp. (Stemonuraceae) & 13.5 \\
\hline 3 & ${ }^{* * S h o r e a ~ c o n t o r t a ~ V i d a l ~(D i p t e r o c a r p a c e a e) ~}$ & 9.7 & & Ficus septica Burm. F. (Moraceae) & 13.5 \\
\hline \multirow[t]{2}{*}{4} & Canarium europhyllum (Burseraceae) & 6.9 & 3 & $\begin{array}{l}\text { Guioa koelreuteria (Blanco) Merr. } \\
\text { (Sapindaceae) }\end{array}$ & 9.6 \\
\hline & $\begin{array}{l}\text { Macaranga tanarius (L.) Muell.-Arg. } \\
\text { (Euphorbiaceae) }\end{array}$ & 6.9 & 4 & Ficus nota (Blanco) Merr. (Moraceae) & 7.7 \\
\hline 5 & $\begin{array}{l}\text { Cleistanthus angustifolius Merr. } \\
\text { (Phyllanthaceae) }\end{array}$ & 5.6 & 5 & $\begin{array}{l}{ }^{* *} \text { Diospyros pilosanthera Blanco } \\
\text { (Ebenaceae) }\end{array}$ & 5.8 \\
\hline \multirow[t]{3}{*}{6} & Barringtonia racemosa (L.) Blume ex DC. & 4.2 & 6 & $\begin{array}{l}\text { Pterospermum celebicum Miq. } \\
\text { (Malvaceae) }\end{array}$ & 3.8 \\
\hline & $\begin{array}{l}{ }^{* * D i o s p y r o s ~ p i l o s a n t h e r a ~ B l a n c o ~} \\
\text { (Ebenaceae) }\end{array}$ & 4.2 & & Miliusa vidalii Sinc. (Annonaceae) & 3.8 \\
\hline & Ficus septica Burm. F. (Moraceae) & 4.2 & & $\begin{array}{l}\text { Hopea plagata (Blanco) Vidal } \\
\text { (Dipterocarpaceae) }\end{array}$ & 3.8 \\
\hline \multirow[t]{5}{*}{7} & $\begin{array}{l}\text { Litsea grandis (Wall. ex Nees) Hook.f. } \\
\text { (Lauraceae) }\end{array}$ & 2.8 & & Kleinhovia hospita L. (Byttneriaceae) & 3.8 \\
\hline & Mischocarpus sp. (Sapindaceae) & 2.8 & 7 & $\begin{array}{l}\text { **Dipterocarpus grandiflorus Blanco } \\
\text { (Dipterocarpaceae) }\end{array}$ & 1.9 \\
\hline & Pterospermum celebicum Miq. (Malvaceae) & 2.8 & & Vitex turczaninowii Merr. (Lamiaceae) & 1.9 \\
\hline & Artocarpus ovatus Blanco (Moraceae) & 2.8 & & $\begin{array}{l}\text { Parkia timoriana (DC.) Merr. } \\
\text { (Fabaceae) }\end{array}$ & 1.9 \\
\hline & $\begin{array}{l}{ }^{* * A r t o c a r p u s ~ b l a n c o i ~(E l m e r) ~ M e r r . ~} \\
\text { (Moraceae) }\end{array}$ & 2.8 & & $\begin{array}{l}\text { Macaranga tanarius (L.) Muell.-Arg. } \\
\text { (Euphorbiaceae) }\end{array}$ & 1.9 \\
\hline \multirow[t]{6}{*}{8} & Cryptocarya acuminata Merr. (Lauraceae) & 1.4 & & $\begin{array}{l}\text { Allophyllus cobbe (L.) Raeusch. } \\
\text { (Sapindaceae) }\end{array}$ & 1.9 \\
\hline & $\begin{array}{l}\text { **Myristica philippinensis Lam. } \\
\text { (Myristicaceae) }\end{array}$ & 1.4 & & $\begin{array}{l}{ }^{*} \text { Anisoptera thurifera (Blanco) Blume } \\
\text { (Dipterocarpaceae) }\end{array}$ & 1.9 \\
\hline & Milleta stipulata Dunn. (Fabaceae) & 1.4 & & $\begin{array}{l}\text { Buchanania arborescens (Blume) } \\
\text { Blume (Anacardiaceae) }\end{array}$ & 1.9 \\
\hline & $\begin{array}{l}\text { Palaquium tenuipetiolatum Merr. } \\
\text { (Sapotaceae) }\end{array}$ & 1.4 & & $\begin{array}{l}\text { Calophyllum inophyllum L. } \\
\text { (Calophyllaceae) }\end{array}$ & 1.9 \\
\hline & Grewia multiflora Juss. (Sparmanniaceae) & 1.4 & & & \\
\hline & $\begin{array}{l}{ }^{*} \text { Anisoptera thurifera (Blanco) Blume } \\
\text { (Dipterocarpaceae) }\end{array}$ & 1.4 & & & \\
\hline
\end{tabular}


Philippine Journal of Systematic Biology | Galias D.C. and V.C. Cuevas: The Regenerating Forest of Magbukún Aeta

\begin{tabular}{|c|c|c|c|c|c|}
\hline \multicolumn{3}{|c|}{ SITE 1 (HULO) } & \multicolumn{3}{|c|}{ SITE 2 (BUOD) } \\
\hline Rank & Species/ Family & $(\%)$ & Rank & Species/ Family & $(\%)$ \\
\hline & $\begin{array}{l}\text { Tabernaemontana pandacaqui Lam. } \\
\text { (Apocynaceae) }\end{array}$ & 1.4 & & & \\
\hline & Calophyllum inophyllum L. (Calophyllaceae) & 1.4 & & & \\
\hline & Elattostachys sp. (Sapindaceae) & 1.4 & & & \\
\hline & Bischofia javanica Blume (Phyllantaceae) & 1.4 & & & \\
\hline & Ficus odorata (Blanco) Merr. (Moraceae) & 1.4 & & & \\
\hline
\end{tabular}

** Threatened species included in The IUCN Red List of Threatened Species 2017-3 and/or DAO No. 2017-11. 
Philippine Journal of Systematic Biology | Galias D.C. and V.C. Cuevas: The Regenerating Forest of Magbukún Aeta

Table 4. Co-dominant (pole trees; $\mathrm{DBH}>10-20 \mathrm{~cm}$ ) encountered within the forest of KNRA.

\begin{tabular}{|c|c|c|c|c|c|}
\hline \multicolumn{3}{|c|}{ SITE 1 (HULO) } & \multicolumn{3}{|c|}{ SITE 2 (BUOD) } \\
\hline Rank & Species/ Family & $(\%)$ & Rank & Species/ Family & $(\%)$ \\
\hline \multirow[t]{2}{*}{1} & $\begin{array}{l}\text { Macaranga tanarius (L.) Muell.-Arg. } \\
\text { (Euphorbiaceae) }\end{array}$ & 25.9 & 1 & $\begin{array}{l}{ }^{* *} \text { Shorea guiso (Blanco) Blume } \\
\text { (Dipterocarpaceae) }\end{array}$ & 8.3 \\
\hline & & & & $\begin{array}{l}{ }^{* *} \text { Canarium luzonicum (Blume) A. } \\
\text { Gray (Burseraceae) }\end{array}$ & 8.3 \\
\hline 2 & Ficus nota (Blanco) Merr. (Moraceae) & 11.1 & & $\begin{array}{l}\text { Litsea glutinosa (Lour.) C.B. Rob. } \\
\text { (Lauraceae) }\end{array}$ & 8.3 \\
\hline \multirow[t]{2}{*}{3} & Ficus odorata (Blanco) Merr.(Moraceae) & 9.3 & & Ficus nota (Blanco) Merr. (Moraceae) & 8.3 \\
\hline & Ficus septica Burm. F.(Moraceae) & 9.3 & & & \\
\hline \multirow[t]{2}{*}{4} & $\begin{array}{l}{ }^{* *} \text { Artocarpus blancoi (Elmer) Merr. } \\
\text { (Moraceae) }\end{array}$ & 5.6 & & & \\
\hline & & & 2 & $\begin{array}{l}\text { Kanapia monstrosa (A. Rich.) Arriola \& } \\
\text { Alejandro (Rubiaceae) }\end{array}$ & 4.2 \\
\hline \multirow[t]{5}{*}{5} & $\begin{array}{l}\text { **Macaranga grandiflora (Blanco) Merr. } \\
\text { (Euphorbiaceae) }\end{array}$ & 3.7 & & $\begin{array}{l}\text { Diospyros pilosanthera Blanco } \\
\text { (Ebenaceae) }\end{array}$ & 4.2 \\
\hline & $\begin{array}{l}\text { Lagerstroemia speciosa (L.) Pers. } \\
\text { (Lythraceae) }\end{array}$ & 3.7 & & Fahrenheitia sp. (Euphorbiaceae) & 4.2 \\
\hline & $\begin{array}{l}\text { Litsea grandis (Wall. ex Nees) Hook.f. } \\
\text { (Lauraceae) }\end{array}$ & 3.7 & & $\begin{array}{l}\text { Ficus odorata (Blanco) Merr. } \\
\text { (Moraceae) }\end{array}$ & 4.2 \\
\hline & Litsea sp. (Lauraceae) & 3.7 & & Ficus septica Burm. f. (Moraceae) & 4.2 \\
\hline & & & & Gomphandra sp. & 4.2 \\
\hline \multirow[t]{9}{*}{6} & $\begin{array}{l}\text { Morinda citrifolia L. f. bracteata (Roxb.) } \\
\text { Hook.f. (Rubiaceae) }\end{array}$ & 1.9 & & $\begin{array}{l}\text { Guioa koelreuteria (Blanco) Merr. } \\
\text { (Sapindaceae) }\end{array}$ & 4.2 \\
\hline & $\begin{array}{l}\text { *Afzelia rhomboidea (Blanco) Vidal } \\
\text { (Fabaceae) }\end{array}$ & 1.9 & & $\begin{array}{l}\text { Heritiera sylvatica Vidal } \\
\text { (Sterculiaceae) }\end{array}$ & 4.2 \\
\hline & Bombax ceiba L. (Bombacaceae) & 1.9 & & $\begin{array}{l}\text { Litsea grandis (Wall. ex Nees) Hook.f. } \\
\text { (Lauraceae) }\end{array}$ & 4.2 \\
\hline & $\begin{array}{l}\text { **Dipterocarpus gracilis Blume } \\
\text { (Dipterocarpaceae) }\end{array}$ & 1.9 & & $\begin{array}{l}\text { **Mangifera altissima Blanco } \\
\text { (Anacardiaceae) }\end{array}$ & 4.2 \\
\hline & $\begin{array}{l}{ }^{*} \text { Koordersiodendron pinnatum (Blanco) } \\
\text { Merr. (Anacardiaceae) }\end{array}$ & 1.9 & & Miliusa vidalii Sinc. (Annonaceae) & 4.2 \\
\hline & $\begin{array}{l}\text { Xanthophyllum flavescens Roxb. } \\
\text { (Polygalaceae) }\end{array}$ & 1.9 & & $\begin{array}{l}\text { Palaquium tenuipetiolatum Merr. } \\
\text { (Sapotaceae) }\end{array}$ & 4.2 \\
\hline & Alstonia scholaris (L.) R. Br. (Apocynaceae) & 1.9 & & $\begin{array}{l}\text { Pterospermum celebicum Miq. } \\
\text { (Malvaceae) }\end{array}$ & 4.2 \\
\hline & $\begin{array}{l}\text { Shorea guiso (Blanco) Blume } \\
\text { (Dipterocarpaceae) }\end{array}$ & 1.9 & & $\begin{array}{l}{ }^{* * *} \text { Shorea contorta Vidal } \\
\text { (Dipterocarpaceae) }\end{array}$ & 4.2 \\
\hline & & & & Syzygium sp. (Myrtaceae) & 4.2 \\
\hline
\end{tabular}




\section{SITE 1 (HULO)}

\begin{tabular}{|c|c|c|}
\hline Rank & Species/ Family & $(\%)$ \\
\hline & Pterospermum celebicum Miq. (Malvaceae) & 1.9 \\
\hline & $\begin{array}{l}\text { Maranthes corymbosa Blume } \\
\text { (Chrysobalanaceae) }\end{array}$ & 1.9 \\
\hline & $\begin{array}{l}{ }^{*} \text { Semecarpus paucinervia Merr. } \\
\text { (Anacardiaceae) }\end{array}$ & 1.9 \\
\hline & $\begin{array}{l}\text { Wendlandia philippinensis Cowan } \\
\text { (Rubiaceae) }\end{array}$ & 1.9 \\
\hline & $\begin{array}{l}\text { Litsea glutinosa (Lour.) C.B. Rob. } \\
\text { (Lauraceae) }\end{array}$ & 1.9 \\
\hline
\end{tabular}

**Threatened species included in The IUCN Red List of Threatened Species 2017-3 and/or DAO No. 2017-11. 
Philippine Journal of Systematic Biology | Galias D.C. and V.C. Cuevas: The Regenerating Forest of Magbukún Aeta

Table 5. Dominant (standard trees; DBH $\geq 20 \mathrm{~cm}$ ) encountered within the forest of KNRA.

\begin{tabular}{|c|c|c|c|c|c|}
\hline \multicolumn{3}{|c|}{ SITE 1 (HULO) } & \multicolumn{3}{|c|}{ SITE 2 (BUOD) } \\
\hline Rank & Species/ Family & $(\%)$ & Rank & Species/ Family & $(\%)$ \\
\hline 1 & ${ }^{* * *}$ Shorea contorta Vidal (Dipterocarpaceae) & 20.0 & 1 & $\begin{array}{l}{ }^{* * H o p e a ~ p l a g a t a ~(B l a n c o) ~ V i d a l ~} \\
\text { (Dipterocarpaceae) }\end{array}$ & 17.5 \\
\hline 2 & Canarium europhyllum Perkins (Burseraceae) & 16.7 & 2 & $\begin{array}{l}\text { **Diospyros pilosanthera Blanco } \\
\text { (Ebenaceae) }\end{array}$ & 15.0 \\
\hline 3 & Bombax ceiba L. (Bombacaceae) & 10.0 & & $\begin{array}{l}{ }^{* * *} \text { Shorea contorta Vidal } \\
\text { (Dipterocarpaceae) }\end{array}$ & \\
\hline 4 & $\begin{array}{l}\text { Alseodaphne longipes Quisumbing \& Merr. } \\
\text { (Lauraceae) }\end{array}$ & 3.3 & 3 & Heritiera sy/vatica Vidal (Sterculiaceae) & 7.5 \\
\hline & $\begin{array}{l}\text { Cleistanthus angustifolius Merr. } \\
\text { (Phyllanthaceae) }\end{array}$ & 3.3 & & $\begin{array}{l}{ }^{* *} \text { Anisoptera thurifera (Blanco) Blume } \\
\text { (Dipterocarpaceae) }\end{array}$ & 7.5 \\
\hline & $\begin{array}{l}\text { Pterocymbium tinctorium (Blanco) Merr. } \\
\text { (Sterculiaceae) }\end{array}$ & 3.3 & & $\begin{array}{l}{ }^{* *} \text { Dimocarpus longan ssp. malesianus } \\
\text { Leenh. (Sapindaceae) }\end{array}$ & 7.5 \\
\hline & ${ }^{* \star}$ Trema orientalis (L.) Blume (Cannabaceae) & 3.3 & 4 & $\begin{array}{l}\text { Macaranga tanarius (L.) Muell.-Arg. } \\
\text { (Euphorbiaceae) }\end{array}$ & 5.0 \\
\hline & $\begin{array}{l}\text { **Macaranga grandiflora (Blanco) Merr. } \\
\text { (Euphorbiaceae) }\end{array}$ & 3.3 & & $\begin{array}{l}{ }^{* *} \text { Shorea guiso (Blanco) Blume } \\
\text { (Dipterocarpaceae) }\end{array}$ & 5.0 \\
\hline & Ficus variegata Blume (Moraceae) & 3.3 & & Ficus benjamina L. (Moraceae) & 5.0 \\
\hline & ${ }^{* *}$ Afzelia rhomboidea (Blanco) Vidal (Fabaceae) & 3.3 & 5 & Ficus septica Burm. f. (Moraceae) & 2.5 \\
\hline & $\begin{array}{l}\text { Casearia fuliginosa (Blanco) Blanco } \\
\text { (Salicaceae) }\end{array}$ & 3.3 & & Vitex turczaninowii Merr. (Lamiaceae) & 2.5 \\
\hline & Cynometra sp. (Fabaceae) & 3.3 & & Miliusa vidalii Sinc. (Annonaceae) & 2.5 \\
\hline & Diospyros pilosanthera Blanco (Ebenaceae) & 3.3 & & Bombax ceiba L. (Bombacaceae) & 2.5 \\
\hline & ${ }^{* * M a n g i f e r a ~ a l t i s s i m a ~ B l a n c o ~(A n a c a r d i a c e a e) ~}$ & 3.3 & & Parkia timoriana (DC.) Merr. (Fabaceae) & 2.5 \\
\hline & $\begin{array}{l}{ }^{* *} \text { Anisoptera thurifera (Blanco) Blume } \\
\text { (Dipterocarpaceae) }\end{array}$ & 3.3 & & $\begin{array}{l}\text { Terminalia foetidissima Griff. } \\
\text { (Combretaceae) }\end{array}$ & 2.5 \\
\hline & $\begin{array}{l}{ }^{* *} \text { Dimocarpus longan ssp. malesianus Leenh. } \\
\text { (Sapindaceae) }\end{array}$ & 3.3 & & Milleta stipulata Dunn. (Fabaceae) & 2.5 \\
\hline & Ganophyllum falcatum Blume (Sapindaceae) & 3.3 & & $\begin{array}{l}{ }^{* *} \text { Canarium luzonicum (Blume) A. Gray } \\
\text { (Burseraceae) }\end{array}$ & 2.5 \\
\hline & Ficus subcordata Blume (Moraceae) & 3.3 & & $\begin{array}{l}{ }^{* *} \text { Myristica philippinensis Lam. } \\
\text { (Myristicaceae) }\end{array}$ & 2.5 \\
\hline & Mischocarpus sp. (Sapindaceae) & 3.3 & & $\begin{array}{l}\text { Alseodaphne longipes Quisumbing \& Merr. } \\
\text { (Lauraceae) }\end{array}$ & 2.5 \\
\hline
\end{tabular}

**Threatened species included in The IUCN Red List of Threatened Species 2017-3 and/or DAO No. 2017-11. 
previously a dipterocarp forest. One possible reason why there are still large dipterocarp trees in this site would be the steep terrain in this forest patch. According to some Aeta elders, this topographic constraint is the reason why loggers were not able to harvest these big trees. This statement was supported by Langenberger (2006) and according to him dipterocarp forest remnants are now generally found in localities where large-scale logging was not profitable and where access was hampered by the difficult terrain as in rugged and mountainous areas.

\section{Vegetation Structure}

Species dominance. Overall, the top five species with highest IP included Shorea contorta Vidal (white lauan; Dipterocarpaceae) (IP=9.731) followed by Macaranga tanarius (L.) Muell.-Arg. (binunga; Euphorbiaceae) (IP=5.659), Canarium europhyllum (kila; Burseraceae), (IP=4.538), Ficus septica Burm. F. (hauili; Moraceae) $(\mathrm{IP}=4.450)$ and Ficus nota (Blanco) Merr. (tibig; Moraceae) (IP=4.416). The dominance of white lauan was attributed to its high density (125 trees/ha) and high dominance (TBA $\left.=49,797 \mathrm{~cm}^{2}\right)$. Nonetheless, out of the 29 white lauan individuals listed, about $55 \%$ are seedlings (DBH $\geq 10 \mathrm{~cm}$, mean $=3.9 \mathrm{~cm}$ ), and only four individuals have a $100 \mathrm{~cm}$ and bigger $\mathrm{DBH}$. From this we can infer that most of them were still small trees, and are just starting to be established in the area. Interestingly two other dipterocarps were among the top 10 most dominant trees; these were Anisoptera thurifera (Blanco) Blume (ranked $6^{\text {th }}$ ) and Hopea plagata (Blanco) Vidal (ranked $8^{\text {th }}$ ). Moreover, close behind was Shorea guiso (Blanco) Blume, another dipterocarp and ranked $11^{\text {th }}$.

Although Macaranga tanarius (L.) Muell.-Arg. (binunga) has the highest frequency (appeared 13 out of 60 quadrats), it only ranked second. This was mainly because of its low density (94 trees/ha) and low dominance (TBA=12,736). Ficus subcordata Blume (payapa) has the highest mean basal area $\left(14,920 \mathrm{~cm}^{2}\right)$ in this site, from the lone large individual of payapa tree recorded.

Species diversity. Using Shannon-Wiener's Index $(H)$ and Simpson's Index $(D)$ formulae, very high values for species diversity were observed in this site $\left(H^{\prime}=3.817 ; D^{\prime}=0.968\right)$ (Table 6). This very high diversity can be the result of a very high Index of Evenness ( $\mathcal{J}$ (0.893) and very low Dominance (D) (0.044). This only means that the trees in this site were evenly distributed and there were no dominating tree species. Fernando and co-workers (2008) mentioned that species diversity in a semi-evergreen tropical rain forest is high but less than that in the lowland evergreen forest. These data on species diversity again showed that the remaining forest in the reservation area exhibited the stages of regeneration characteristic of the tropical rainforest where species diversity is very high following major disturbances such as strong typhoons, earthquakes or cutting of large trees that open wide spaces that allow individuals of new species to colonize the open area. Usually the vegetation that develops right after clearing of old-growth forest (i.e., minimal land use intensity) tends to be poorer both in terms of species richness and abundance than that in cleared sites previously supporting successional vegetation (Guariguata and Ostertag, 2001). In terms of species richness, Whitmore (1990) stated that the most species-rich forest will be the one that includes patches of secondary and primary forests. The former is recovering from a big disturbance and consisting of pioneers; while the latter is composed of climax species. After the succession and the whole landscape is primary forest again, there will be no pioneers and total floristic richness will be reduced. Data from this study tend to support the statement of Whitmore (1990) on high species richness of secondary forest.

\section{The strong need for protection and conservation of this remaining KNRA Forest}

The study area is part of Morong, Bataan and located inside the Bataan Natural Park (BNP) which is one of the Key Biodiversity Areas (KBA) in the Philippines. According to the KBA booklet (Conservation International Philippines, DENRPAWB and Haribon Foundation, n.d.), there are 21 Trigger Species in the area, which include one (1) Endangered Species (EN), five (5) Vulnerable Species (VU) and 15 Restricted Range Species (RR).

Rechecking the IUCN Red List (2017-3) and DAO No. 201711 , it was found out that one species was categorized as Critically Endangered (IUCN) and one species listed in DAO 2017-11 as Endangered Species (Category B) (Tables 7 \& 8).

Shorea contorta Vidal (Dipterocarpaceae) is categorized as Critically Endangered Species in the IUCN Red List (2017-3) along with eight other species listed as Vulnerable. Furthermore, Dimocarpus longan Lour. ssp. malesianus Leenh. (Sapindaceae) is already considered as near threatened species. Checking the DAO 2017-11, it was found out that Afzelia rhomboidea (Blanco) Vidal (Fabaceae) is considered as Endangered Species (Category B). Eleven more tree species are under Category $C$ considered as Vulnerable Species, three are listed as Other Threatened Species and one tree species, Dimocarpus longan Lour. ssp. malesianus Leenh. (Sapindaceae) is already under Near Threatened status.

The forest is also home to various endemic species such as Artocarpus blancoi (Elmer) Merr. (Moraceae), A.. ovatus 
Table 6. Comparison of the computed values of indices in the forest of KNRA

\begin{tabular}{lcccc}
\hline & $\begin{array}{c}\text { Site 1 } \\
\text { (Hulo) }\end{array}$ & $\begin{array}{c}\text { Site 2 } \\
\text { (Buod) }\end{array}$ & Overall & Mean \\
\hline NO. OF INDIVIDUALS & 238 & 160 & 398 \\
\hline Species richness (s) & 57 & 46 & 78 & 1,407 \\
Trees per hectare & 1,243 & 1,624 & 1,355 & 2.678 \\
Mean distance (m/tree) & 2.836 & 2.482 & 2.717 & 3.616 \\
\hline TREE DIVERSITY INDICES & & & 3.817 & 0.961 \\
\hline Shannon Index of Diversity $(H)$ & 3.593 & 3.439 & 0.968 & 0.039 \\
Simpson Index of Diversity $(D)$ & 0.958 & 0.956 & 0.032 & 0.878 \\
Simpson Index of Dominance $(D)$ & 0.042 & 0.044 & 0.874 & \\
Pielou's Index of Evenness $(J)$ & 0.867 & 0.893 & & \\
\hline
\end{tabular}

Table 7. Threatened species listed in The IUCN Red List of Threatened Species 2017-3.

\begin{tabular}{lll}
\hline \multicolumn{1}{c}{ FAMILY } & \multicolumn{1}{c}{ SPECIES } & \multicolumn{1}{c}{ COMMON NAME } \\
\hline Anacardiaceae & Mangifera altissima Blanco & Pahutan \\
${ }^{* * *}$ Dipterocarpaceae & Shorea contorta Vidal & White Lauan \\
Dipterocarpaceae & Hopea plagata (Blanco) Vidal & Yakal saplungan \\
Dipterocarpaceae & Anisoptera thurifera (Blanco) Blume & Palosapis \\
Dipterocarpaceae & Dipterocarpus gracilis Blume & Panau \\
Dipterocarpaceae & ${ }^{* * D i p t e r o c a r p u s ~ g r a n d i f l o r u s ~ B l a n c o ~}$ & Apitong \\
Dipterocarpaceae & Shorea guiso (Blanco) Blume & Guiso \\
Moraceae & Artocarpus blancoi (Elmer) Merr. & Antipolo \\
Fabaceae & Afzelia rhomboidea (Blanco) Vidal & Tindalo \\
Sapindaceae & ${ }^{*}$ Dimocarpus longan Lour. & Alupag lalaki \\
& ssp.malesianus Leenh & \\
\hline
\end{tabular}

\footnotetext{
${ }^{* * *}$ Critically endangered

${ }^{* *}$ Endangered

*Near Threatened
} 
Table 8. Threatened tree species listed in the Department of Environment and Natural Resources Administrative Order (DAO) No. 2017-11.

FAMILY SPECIES COMMON NAME

\section{Category B: Endangered Species}

Fabaceae

\section{Category C: Vulnerable Species}

Anacardiaceae

Anacardiaceae

Dipterocarpaceae

Dipterocarpaceae

Dipterocarpaceae

Dipterocarpaceae

Dipterocarpaceae

Ebenaceae

Euphorbiaceae

Moraceae

Sapotaceae
Afzelia rhomboidea (Blanco) Vidal

Mangifera altissima Blanco

Semecarpus paucinervia Merr.

Hopea plagata (Blanco) Vidal

Anisoptera thurifera (Blanco) Blume

Dipterocarpus gracilis Blume

Dipterocarpus grandiflorus Blanco

Shorea contorta Vidal

Diospyrus pilosanthera Blanco

Macaranga grandiflora

Artocarpus blancoi (Elmer) Merr.

Palaquim philippense (Perr.) C.B. Rob.
Tindalo

Pahutan

Ligas- ilanan

Yakal saplungan

Palosapis

Panau

Apitong

White Lauan

Bulong-eta

Takip- asin

Antipolo

Malak- malak

\section{Category D: Other Threatened Species}

Anacardiaceae

Burseraceae

Myristicaceae
Koordersiodendron pinnatum (Blanco) Merr. Amugis

Canarium luzonicum (Blume) A. Gray

Myristica philippinensis Lam.
Piling- liitan

Duguan

Table 9. List of endemic tree species encountered within the KNRA Forest

\begin{tabular}{lll}
\hline \multicolumn{1}{c}{ FAMILY } & \multicolumn{1}{c}{ SPECIES } & \multicolumn{1}{c}{ COMMON NAME } \\
\hline Burseraceae & Canarium luzonicum (Blume) A. Gray & Piling liitan \\
Dipterocarpaceae & Shorea contorta Vidal & White lauan \\
Euphorbiaceae & Macaranga grandiflora (Blanco) Merr. & Takip- asin \\
Leeaceae & Leea philippinensis Merr. & Kaliantan \\
Moraceae & Artocarpus blancoi (Elmer) Merr. & Antipolo \\
& Artocarpus ovatus Blanco & Anubing \\
Myristicaceae & Myristica philippinensis Lam. & Duguan \\
Phyllantaceae & Cleistanthus angustifolius Merr. & Aniatan kitid \\
Rubiaceae & Wendlandia philippinensis Cowan & Mamboi \\
Sapotaceae & Palaquim philippense (Perr.) C.B. Rob. & Malak-malak \\
& Palaquim tenuipetiolatum Merr. & Maniknik \\
Stemonuraceae & Gomphandra luzoniensis Merr. subsp. & Mabunot \\
& Iuzoniensis Merr. & \\
\hline
\end{tabular}




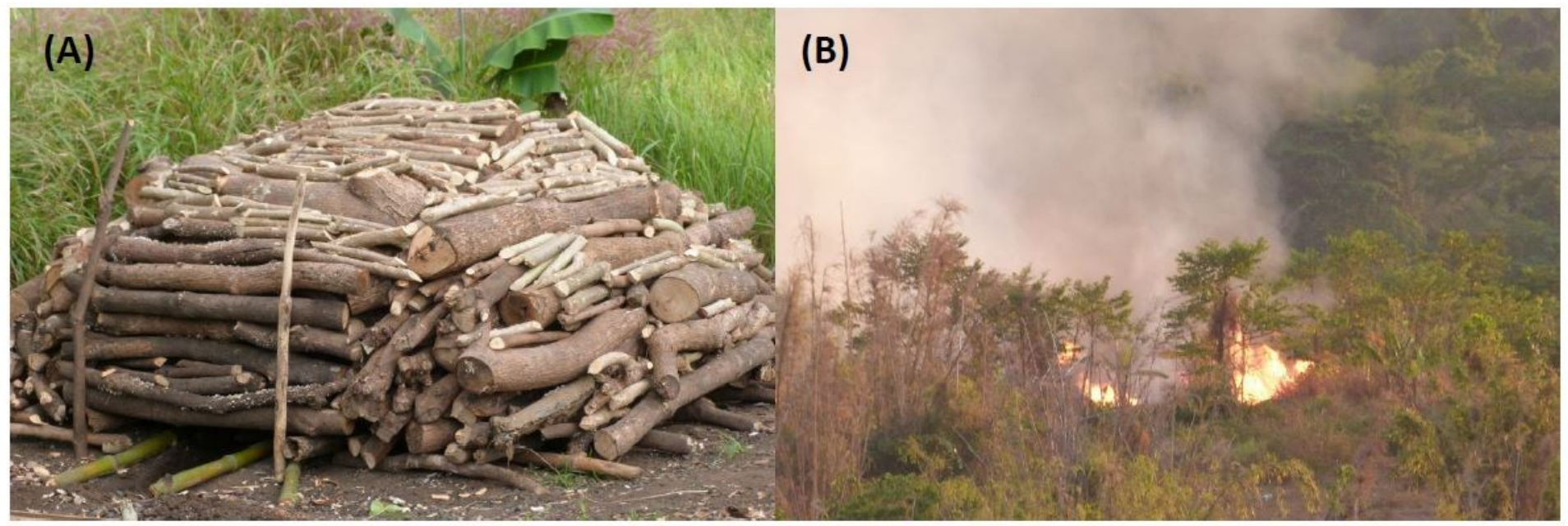

Figure 4. Some of the common forest disturbances in the KNRA: A. Charcoal making, B. Occurrence of fire

Blanco (Moraceae), Macaranga grandiflora (Blanco) Merr. (Euphorbiaceae), Mangifera altissima Blanco (Anacardiaceae), Leea philippinensis Merr. (Leeaceae) and Shorea contorta Vidal (Dipterocarpaceae) among others.

The forest is home to a long list of threatened and endemic species. This only means that the forest is a repository of these species, whose populations are already dwindling or absent in many forests in the country thus, its protection and conservation is a must. Currently, the conservation measures in the area include its designation as an Important Bird Area (IBA), (PH008) and Conservation Priority Act 29 (Conservation International Philippines, DENR- PAWB and Haribon Foundation, n.d.). However, during the study period, activities that are considered as threats to biodiversity were observed such as road development, charcoal making, expansion of agricultural lands and bi-annual grassland fires (see Fig. 4). No quantitative data were collected from these activities since they were outside the focus of the research.

Biodiversity hotspots are areas with exceptional endemism and experiencing habitat loss which brought the country to the brink of ecological ruin (Myers, et al., 2000; Posa, et al., 2008). Based on this definition and the data generated by this study, the remaining forest of KNRA can be considered as a biodiversity hotspot. The original 186 ha KNRA is now only reduced to 27.25 ha, which is home to a number of endemic species. As mentioned, the remaining forest still has good number of species that are now absent in the remaining forests of the country. These species are listed as either critically endangered, endangered or vulnerable species. Protection from fire and expansion of the remaining forest through planting of wildings of native trees in the adjacent brushland therefore is a must. Involving the community in these processes will ensure sustainability.

\section{CONCLUSIONS AND RECOMMENDATIONS}

The data generated in this study showed that the remaining forest of KNRA was once a dipterocarp forest and can now be classified as a young regenerating forest composed primarily of trees in the seedling and sapling stages. The forest is also home to a long list of threatened and endemic species. This only means that the forest serves as a repository of these species, whose populations are already dwindling or absent in many forests in the country; thus its protection and conservation are a must.

\section{ACKNOWLEDGEMENTS}

This study was funded by the Department of Science and Technology-Accelerated Science and Technology Human Resource Development Program-National Science Consortium (DOST-ASTHRDP-NSC) thesis grant to the first author, and the National Academy for Science and Technology (NAST) Hugh Greenwood Environmental Science Research Grant awarded to the second author. This work represents a portion of an MS Botany thesis prepared by the first author and submitted to the Institute of Biological Sciences of the University of the Philippines-Los Baños. This paper is dedicated to the memory of the late Dr. Benito C. Tan, foremost Filipino expert on tropical bryology.

\section{LITERATURE CITED}

Arsenio, J.J., M.P. Medecilo, E.T. Mercado, E.T. Salibay, Jr., and F.A.H. Valera, 2011. The vegetation of Mt. Maculot, Cuenca, Batangas, Philippines. International Journal of Environmental Science and Development, 2(4): 274278.

Balilla, V.S., J. Anwar-Mchenry, M.P. Mchenry, R.M. Parkinson, and D.T. Banal, 2012. Aeta Magbukún of Mariveles: Traditional indigenous forest resource use 
practices and the sustainable economic development challenge in remote Philippine regions. Journal of Sustainable Forestry, 31(7): 687-709.

Bankoff, G., 2007. One island too many: Reappraising the extent of deforestation in the Philippines prior to 1946. Journal of Historical Geography, 33: 314-334.

Bustos, M.D.G., A. Saul, N.P. Salazar and M. Gomes, 1997. Profile of Morong, Bataan, an area of low malaria endemicity in the Philippines. Acta Tropica, 63(4): 195207.

Calderon, M.M., N.C. Bantayan, F.B. Pulhin and L.J. Cruz, 2013. Exploring the Forestry Carbon Finance Potential for an Indigenous Cultural Community in the Philippines. Retrieved from http://www.eepsea.net/pub/rr/2013RR1_Calderon.pdf (accessed 01/23/2014).

Chazdon, R., 2003. Tropical forest recovery: Legacies of human impact and natural disturbances. Perspectives in Plant Ecology, Evolution and Systematics, 6: 51-71.

Chokkalingam, U., A.P. Carandang, J.M. Pulhin, R.D. Lasco and R.J.J. Peras, 2006. One Century of Forest Rehabilitation in the Philippines: Approaches, Outcomes and Lessons. Center for International Forestry Research. Bogor, Indonesia. 132 pp.

Conservation International Philippines, DENR-PAWB And Haribon Foundation. undated. Priority Sites for Conservation in the Philippines: Key Biodiversity Areas.

Cottee-Jones H.E.W., O. Bajpal, L.B. Chaudhary and R.J. Whittaker, 2016. The importance of Ficus (Moraceae) trees for tropical forest restoration. Biotropica, 48: 413419.

Cruz, L.J., 2010. Bioprospecting in the Ancestral Domain of Aytas in Bataan. Powerpoint presentation NAST, UP Diliman, Center for Bio-Molecular Science Foundation.

De Guzman, E.D., R.M. Umali and E.D. Sotalbo. 1986. Guide to Philippine Flora and Fauna. Volume III. Dipterocarp. Non-Dipterocarp. JMC Press. Inc. Quezon City Philippines.

DENR Administrative Order No. 2007- 01. Establishing the National List of Threatened Philippine Plants and their Categories, and the List of other Wildlife Species.

Devi, L.S and P.S Yadava, 2006. Floristic diversity assessment and vegetation analysis of tropical semievergreen forest of Manipur, north east India. Tropical Ecology, 47(1): 89-98.

Diereck, D. and D. Holscher, 2009. Species-specific tree water use characteristics in reforestation stands in the Philippines. Agricultural and Forest Meteorology, 149: 1317-1326. doi:10.1016/j.agrformet.2009.03.003

Fernando, E.S., M.H. Suh, J. Lee and D.K. Lee, 2008. Forest Formations of the Philippines. Korea: ASEAN-Korea Environmental Cooperation Unit, Seoul National University. Seoul: Geobook Publishing Co.
Galang, A.P., S.R. Mesina, M.R. Marciano, A.M. Tungol and I.P. Sarmiento, 2012. Philippine Native Trees 101: Up Close and Personal. Green Convergence for Safe Food, Healthy Environment and Sustainable Economy, and Hortica Filipina Foundation Inc., Manila.

Guariguata, M.R. and R. Ostertag, 2001. Neotropical secondary forest succession: Changes in structural and functional characteristics. Forest Ecology and Management, 148: 185-206.

Lalfakawma, U.K. Sahoo, S. Roy, K. Vanlalhriatpuia and P.C. Vanalalhluna, 2009. Community composition and tree population structure in undisturbed and disturbed tropical semi-evergreen forest stands of North-East India. Applied Ecology and Environmental Research, 7 (4): 303-318.

Langenberger, G, 2006. Habitat distribution of dipterocarp species in the Leyte Cordillera: An indicator for species site suitability in local reforestation programs. Annals of Forest Science, 63(2): 149-156.

Liu, D.S., L.R. Iverson and S. Brown, 1993. Rates and patterns of deforestation in the Philippines: Application of Geographic Information System analysis. Forest Ecology and Management, 57: 1-16.

Motin, B.B, L.J. Cruz, F. Gifford, and M.A. Kron, 2006. A Modified Socio-Cultural Development Index (SCDI) for the Aytas of Kanawan, Morong, Bataan, Philippines. Indigenous Perspectives, 8(1):51- 71.

Mueller-Dombois, D. and H. Ellenberg, 1974. Aims and Methods of Vegetation Ecology. New York, USA: JohnWiley and Sons.

Omeja, P., J. Obua and A.B. Cunningham, 2004. Regeneration, density and size class distribution of tree species used for drum making in central Uganda. African Journal of Ecology, 42: 129-136.

Outoma, J., G. Ouma, D. Okeyo and B. Anyango, 2014. Species composition and stand structure of secondary and plantation Forest in a Kenyan rainforest. Journal of Horticulture and Forestry, 6(4): 38-49.

Pelser, P.B., J.F. Barcelona and D.L. Nickrent (eds). 2011 onwards. Co's Digital Flora of the Philippines, URL: http://www.philippineplants.org. (accessed: April 2018).

Philippine Tropical Forest Conservation Foundation, Inc., 2013. Connecting Forest, People and Development: Lessons and Insights from 10 Years of the Tropical Forest Conservation Fund in the Philippines. Aptissimi Development Innovations, Inc. pp. 80- 91.

Rasingam L. and N. Parathasarathy, 2009. Tree species diversity and population structure across major forest formations and disturbance categories in Little Andaman Island, India. Tropical Ecology, 50(1): 89-102.

Sarkar, M. and A. Devi, 2014. Assessment of diversity, population structure and regeneration status of tree species in Hollongapar Gibbon Wildlife Sanctuary, 
Philippine Journal of Systematic Biology | Galias D.C. and V.C. Cuevas: The Regenerating Forest of Magbukún Aeta Assam, northeast India. Tropical Plant Research, 1(2): 26 -36 .

Shanahan, M., S. So, S.G. Compton and R. Corlett. 2001. Fig -eating by vertebrate frugivores: A global review. Biological Reviews, 76: 529-572.

The IUCN Red List Of Threatened Species 2017-3. URL: www.iucnredlist.org. (accessed: April 2018).

Whitford, H.N., 1911. The Forest of the Philippines. Part I, Forest Types and Products. Bureau of Printing. Manila.

Whitmore, T.C., 1990. An Introduction to Tropical Rain Forests. Claredon Press. Oxford. 